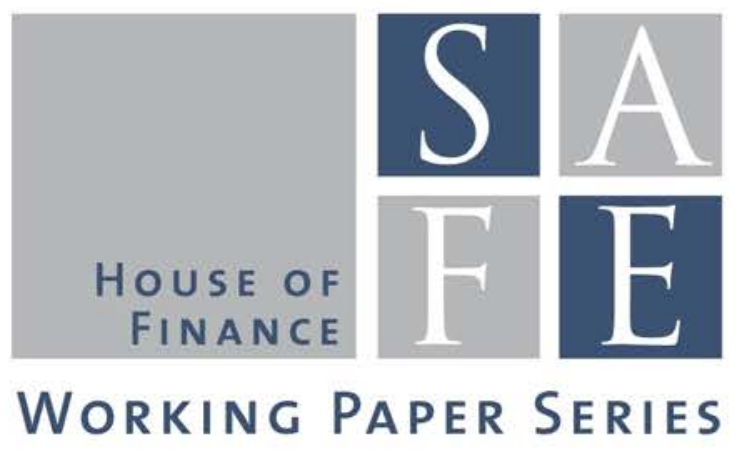

Nicole Branger - Christian Schlag - Lue Wu

\title{
'Nobody is Perfect': Asset Pricing and Long-Run Survival When Heterogeneous Investors Exhibit Different Kinds of Filtering Errors
}

SAFE Working Paper No. 114

SAFE I Sustainable Architecture for Finance in Europe A cooperation of the Center for Financial Studies and Goethe University Frankfurt 


\section{Non-Technical Summary}

Some quantities on financial markets are unobservable and market participants have to estimate them based on the data they observe. One example is the currently expected growth rate of the economy over the next period, e.g., the next year. This number is not directly observable and varies over time. However, investors can use past data and 'filter' their estimate for the growth rate, which is then the basis of economic decisions (e.g., consumption, saving, asset allocation). The result of this filtering process, however, depends on the initial assumptions made by the investor on the dynamics of the growth rate and its relation to observable quantities. Since the true growth rate is not observable, the investor can never be sure to actually use the 'true' model. In reality investors will furthermore be heterogeneous with respect to the assumptions they make, and in general no investor will have a model that is exactly equal to the true one.

In this paper we analyze the equilibrium implications for expected returns on a stock and for investors' long-run wealth shares in the economy when their models feature different kinds of errors. Investor heterogeneity is represented in a stylized fashion via two investor types, which differ in estimation models they rely on. The quantity they have to estimate is the expected growth rate of aggregate output, i.e., loosely speaking expected economic growth.

The concrete questions we are tackling in the paper are the following: First, is there a mistake on the part of one investor, which is so bad that the investor will (slowly) lose her wealth, no matter what kind of error the other investor commits? Second, is there a direct link between the issue of 'long-run survival' (i.e., if an investor does or does not lose all her wealth over time) and asset pricing results in the sense of expected stock returns and volatilities?

The answer to the first question is 'yes'. For example, when one investor assigns substantially too much informational value to a signal, which in reality just represents noise (a situation sometimes labeled as 'overconfidence'), she will ultimately lose all her wealth, irrespective of the degree to which the other investor assumes, e.g., an incorrect volatility or incorrect long-run mean of the expected growth rate of the economy.

The answer to the second question is 'no' in the sense that the asset pricing results are basically independent from the outcome with respect to long-run survival. For example, in scenarios when the investors are more similar with respect to their long-run share in the economy, return volatilities can nevertheless be higher than in cases when they are very different.

The paper provides insights into the link between (imperfect) information processing by investors and financial market outcomes. It gives an example for a situation characterized by market dynamics, which seem to exhibit features like excess volatility, but when all the elements of the economic decision problem are taken into account, prices and returns are perfectly in line with investor rationality. 


\title{
'Nobody is Perfect': \\ Asset Pricing and Long-Run Survival When Heterogeneous Investors Exhibit Different Kinds of Filtering Errors ${ }^{\text {ht }}$
}

\author{
Nicole Branger ${ }^{\mathrm{a}}$, Christian Schlag ${ }^{\mathrm{b}, *}$, Lue $_{\mathrm{Wu}}^{\mathrm{c}}$ \\ ${ }^{a}$ Finance Center Muenster, University of Muenster, Universitätsstr. 14-16, D-48143 Münster, \\ Germany, phone: +49 (251) 83 22033, fax: +49 (251) 83 22882, e-mail: \\ Nicole.Branger@wiwi.uni-muenster.de \\ ${ }^{b}$ House of Finance, Goethe University Frankfurt, Theodor-W.-Adorno-Platz 3, D-60323 Frankfurt \\ am Main, Germany, phone: +49 (69) 798 33699, fax: +49 (69) 798 33901, e-mail: \\ schlag@finance.uni-frankfurt.de \\ ${ }^{c}$ Oliver Wyman, Friedrich-Ebert-Anlage 49, D-60308 Frankfurt am Main, Germany, phone: +49 \\ (69) 971730, fax:+49 (69) 9717310, e-mail: lue.wu@oliverwyman.com
}

\begin{abstract}
In this paper we analyze an economy with two heterogeneous investors who both exhibit misspecified filtering models for the unobservable expected growth rate of the aggregated dividend. A key result of our analysis with respect to long-run investor survival is that there are degrees of model misspecification on the part of one investor for which there is no compensation by the other investor's deficiency. The main finding with respect to the asset pricing properties of our model is that the two dimensions of asset pricing and survival are basically independent. In scenarios when the investors are more similar with respect to their expected consumption shares, return volatilities can nevertheless be higher than in cases when they are very different.
\end{abstract}

Keywords: General Equilibrium, Asset Allocation, Learning, Different Beliefs, Over-Confidence

JEL: G11, G12

\footnotetext{
${ }^{\star}$ Part of this research was done when Wu was a doctoral student in the graduate program 'Finance and Monetary Economics' at Goethe University Frankfurt. Financial support by Deutsche Forschungsgemeinschaft (DFG) is gratefully acknowledged. Schlag gratefully acknowledges research support from the Research Center SAFE, funded by the State of Hessen initiative for research LOEWE.

${ }^{*}$ Corresponding author
} 


\section{Introduction}

Investor heterogeneity is a key ingredient to modern asset pricing models, since it can help to mitigate or even solve well-known puzzles, be it by generating an equity premium close to the value observed in the data in models even with standard CRRA preferences or by generating a volatility of stock returns which exceeds the volatility of fundamentals like dividends (see, e.g., Dumas et al. (2009)). Besides the asset pricing implications of heterogeneity, a second key issue in such models is the survival of the different investors. As pointed out by Borovicka (2015) and Easley and Yang (2013) the question whether one or more investors survive is generally important from a modeling perspective, since we want to know what the economy looks like in its steady state, i.e., when the initial conditions no longer have an effect on quantities like expected returns or return volatilities.

In this paper we analyze the implications of heterogeneous beliefs. Our two investors hold different beliefs about the true dynamics of an unobservable quantity, which is in our case the expected dividend growth rate. They both use an incorrect model and thus in our economy 'nobody is perfect'. The distinctive feature of our setup is that the errors made by the investor can be with respect to rather different characteristics of the model, so that the magnitude of the errors is not easily and directly comparable. The first question we analyze is whether and to which degree errors made by the two investors can offset each other when it comes to survival. The 
second question we are interested in is whether the impact of errors on volatilities and expected excess returns is additive in a sense, or whether errors of the two investors can again somehow offset each other.

The classical kind of model mis-specification analyzed, e.g., in Scheinkman and Xiong (2003) and Dumas et al. (2009) is 'over-confidence' with respect to a signal that investors can observe in addition to the dividend flow. In this typical setup one investor assumes that innovations to the signal are correlated with innovations to the expected growth rate, whereas the other one correctly assumes that the two innovations are independent, so that the former will disappear from the economy. It is intuitively clear that in such a setup only the rational investor will survive, while the other investor makes consumption and investment decisions which will drive him out of the economy in the long run. This is one of the key results of papers like Dumas et al. (2009), Yan (2008), or Kogan et al. (2006). Yan (2008) also analyzes a model in which both investors can potentially be wrong with respect to the (constant) long-run growth rate, and shows that this basically puts the investor with the larger absolute error at a disadvantage.

Given the literature on models where exactly one of the two agents is at a disadvantage concerning her filtering model or where both investors make the same kind of mistake, we ask the next natural question: What happens when both investors use a mis-specified model to draw their inference about the unobservable expected dividend growth rate, where in the general case the mis-specification will 
be with respect to different parameters? How do these errors trade off against each other? For example, while one investor might be over-confident, the other one might assume an incorrect value for the volatility of the expected growth rate process similar to the specification in Buraschi et al. (2014). Alternatively, one investor might be a long-run optimist or pessimist, i.e., she over- or underestimates the long-run mean of the expected growth rate relative to the true model, while the other investor incorrectly assumes that the innovation in the dividend is correlated with the innovation in the expected growth rate. The model we are going to analyze in this paper is completely general in the sense that both the dividend itself and the signal can be correlated with the expected growth rate process under the true model and/or under the two investors' subjective models.

In contrast to the case when only one investor uses an improper filtering model it is in our setup not a priori clear how different types of errors aggregate to the overall result. The question is, e.g., whether an error with respect to the correlation between signal innovations and the expected growth rate is 'more or less severe' than an improper belief about the long-run mean of the expected growth rate, or, put differently, what the trade-off is between these two types of errors.

Our metric for survival is the equilibrium expected share of total consumption an investor retains over longer and longer time horizons. In case this expectation is increasing we take this as an indication of survival. Note that the expectation is calculated under the objective measure, since we are interested in the true steady 
state, but not in the investor's beliefs about this steady state. Under his subjective measure, an investor always thinks that she will survive. This also explains why it does not occur to the investor who is using the 'worse' model that she is at a disadvantage. Given their respective models, in which they put in a sense infinite trust, both investors behave optimally and rationally and as long as, technically speaking, the investors' subjective probability measures are equivalent 1 they will not know for sure in finite time who (if any) of them is using the correct or at least a better model than the respective counterpart. This only becomes clear in the limit when time goes to infinity leading to long-run survival or extinction, respectively.

To analyze survival we therefore compute the expectation of the consumption share under the true measure for different horizons into the future to see whether there is a tendency towards the concentration of consumption on one investor only. Since in models with preferences exhibiting constant relative risk aversion the simultaneous long-run survival of both investors is a knife-edge case, it will basically always happen that the expected consumption share for one investor moves towards one, but in case both investors simultaneously commit errors it is a priori not clear for which one.

The second dimension of our analysis are the asset pricing implications of our

\footnotetext{
${ }^{1}$ Two probability measures are called equivalent, when their null sets coincide. In our case this means that there is no event which has a non-zero probability under one investor's measure, but a zero probability under the other's.
} 
setup where nobody is perfect. The fundamental question here is whether the fact that not only one but both investors have an improper filtering model increases or decreases asset return volatilities and expected excess returns. On the one hand, the fact that the second investor is no longer perfect obviously diminishes the first investor's disadvantage in terms of survival, and the fact that the investors are now less different with respect to survival might also make asset return volatilities go down. On the other hand, additional filtering errors mean additional 'noise' in the market, so that they might lead to even higher volatilities than before when only one investor was using an incorrect model. Here we again take the perspective of an outside observer by computing all the moments under the objective measure, i.e., we calculate expected returns and volatilities like a researcher given the time series of prices generated by our model.

Before going into the details of the model and the analysis, we want to briefly describe our main findings. In terms of survival it is intuitively clear that there is 'continuity' in some sense, i.e., starting from a situation where investor $i$ uses the true model, whereas investor $j$ does not, and moving to case when investor $i$ commits larger and larger errors, investor $i$ will initially still survive, until a critical value for her own error is reached. The knife-edge case of equal consumption shares over the long run is reached, when these errors exactly compensate each other. Afterwards it will be investor $j$ surviving.

For example, for our parametrization, when investor 2 is overconfident with 
respect to the signal by assuming a correlation of 0.3 between the signal and the expected growth rate (whereas the true correlation is zero), then investor 1 would have to assume an incorrect volatility of the expected growth rate process which deviates by roughly 1.2 percentage points from its true value of 0.03 in order for investor 2 to retain a non-zero expected consumption share in the long run. The more important point here is, however, that there are values for investor 2's degree of signal overconfidence, which can no longer be compensated by investor 1's potentially incorrect volatility specification. So there are limits to the trade-off of different types of errors against each other.

Investor 1 may also commit an error with respect to his assumptions about the drift of the expected growth rate. These kinds of errors seem to be less severe than overconfidence with respect to the signal. For example, the error committed by investor 1 with respect to the long-run mean of the expected growth rate must be substantial relative to the true value to offset investor 2's erroneous specification of a non-zero correlation between signal and expected dividend growth. Assuming again a degree of signal overconfidence of 0.3 , investor 1 would have to assume a long.run expected growth rate of dividends more than twice as high as the true value. Similarly, albeit to a somewhat lesser degree, errors concerning the speed of mean reversion in the expected growth rate process must be rather pronounced relative to the true value to offset a certain degree of signal overconfidence on investor 2's part. With this overconfidence again at 0.3 , investor 1 would have to mis-specify the 
speed of mean reversion to be roughly $50 \%$ higher than the true value.

We can also compare signal overconfidence and dividend overconfidence, i.e., situations in which the investor assumes an incorrect correlation between the expected growth rate and either the signal or the dividend. From a modeling perspective it is then of interest which of these two kinds of overconfidence is the more severe one. We find that signal overconfidence is more harmful to the investor than dividend overconfidence, in the sense that it takes in many cases a larger mistake of the other investor to offset a given level of signal overconfidence than it takes to offset the same level of dividend overconfidence.

Concerning the asset pricing implications of our setup the main message is that there is no simple relationship between the degree to which investors are different with respect to their long-run expected consumption shares and the asset pricing results in terms of volatilities and expected excess returns. There are many cases when a reduction of one investor's disadvantage in terms of survival due to simultaneous model misspecification on the part of the other investor makes stock returns more and not less volatile. Stated differently, it is the absolute value of each model mis-specification which matters for asset pricing moments, not the relative size of the error as compared to the other investor. While there is thus a trade-off between errors when it comes to survival, the errors basically add up when it comes to asset pricing moments. This shows that the long-term and the short-term properties of an asset pricing model cannot deduced from each other in a simple and straightforward 
fashion, but rather represent more or less independent dimensions.

The remainder of the paper is organized as follows. The model setup as well as the filtering equations used by the investors and the equilibrium are given in Section 2. Section 3 gives the results of the numerical analysis for survival and asset pricing. Section 4 concludes.

\section{Model}

\subsection{Basic setup}

We first present the overall setup of the model with a more qualitative discussion of its features. The technical details about the investors' filtering will follow in the next subsection.

The exogenous dividend, or aggregate supply, process in the economy $D_{t}$ follows the process

$$
\frac{d D_{t}}{D_{t}}=\mu_{t} d t+\sigma_{D} d W_{D, t} .
$$

The investors furthermore observe a signal $s$ with dynamics

$$
d s_{t}=\sigma_{s} d W_{s, t} .
$$

The unobservable drift rate of the dividend process $\mu_{t}$ is assumed to follow an Ornstein-Uhlenbeck process:

$$
d \mu_{t}=\kappa\left(\bar{\mu}-\mu_{t}\right) d t+\sigma_{\mu}\left(\alpha_{D} d W_{D, t}+\alpha_{s} d W_{s, t}+\alpha_{\mu} d W_{\mu, t}\right)
$$


$\left(W_{D}, W_{s}, W_{\mu}\right)^{\prime}$ is a three-dimensional standard Wiener process, i.e., the components are independent. We assume $\alpha_{D}^{2}+\alpha_{s}^{2}+\alpha_{\mu}^{2}=1$, so that the quadratic variation of $\mu$ is equal to $\sigma_{\mu}^{2}:^{2}$ The parameters $\alpha_{D}, \alpha_{s}$, and $\alpha_{\mu}$ represent the respective correlation between the expected growth rate and the other sources of randomness in the economy. Under the true model we set $\alpha_{D}=\alpha_{s}=0$, i.e., the dynamics of $\mu$ are not correlated with either the dividend or the signal. When we later analyze the consequences of heterogeneous beliefs, investors can disagree about the information content of both dividend innovations and the signal.

Since $\mu$ is unobservable, investors have to form expectations about it using the observables $D$ and $s$. The key fact that will be exploited for the analysis in this paper is that there is nothing that prevents investors in this economy from assuming different models for the dynamics of $\mu$ in their estimation, as long as the investors' subjective probability measures are equivalent.$^{3}$ These differences in beliefs about the dynamics of the economy can be rather general, i.e., the investors' subjective models can differ with respect to any parameter related to the dynamics of $\mu_{t}$ in (3),

\footnotetext{
${ }^{2}$ Note that the condition $\alpha_{D}^{2}+\alpha_{s}^{2}+\alpha_{\mu}^{2}=1$ implies that the $\alpha$ 's are all less than or equal to 1 in absolute value.

${ }^{3}$ The subjective probability measures are defined on a filtered probability space where the filtration is generated by signal and dividend observations. In contrast, the true measure is defined an a filtered probability space where the filtration is generated by the signal, the dividend, and the expected growth rate.
} 
i.e., with respect to $\kappa, \bar{\mu}, \sigma_{\mu}$, and all the correlation coefficients $\alpha_{D}, \alpha_{s}$, and $\alpha_{\mu}{ }^{4}$

In particular, investor $i=1,2$ assumes the following dynamics of the economy:

$$
\begin{aligned}
\frac{d D_{t}}{D_{t}} & =\mu_{t} d t+\sigma_{D} d W_{D, t, i} \\
d \mu_{t} & =\kappa_{i}\left(\bar{\mu}_{i}-\mu_{t}\right) d t+\sigma_{\mu, i}\left(\alpha_{D, i} d W_{D, t, i}+\alpha_{s, i} d W_{s, t}+\alpha_{\mu, i} d W_{\mu, t, i}\right) \\
d s_{t} & =\sigma_{s} d W_{s, t}
\end{aligned}
$$

again with $\alpha_{s, i}^{2}+\alpha_{\mu, i}^{2}+\alpha_{D, i}^{2}=1$. Note that the Wiener processes $W_{D}$ and $W_{\mu}$ now carry an index $i$, since they are given under investor $i$ 's subjective probability measure $\mathbb{P}^{i}$, while $W_{s}$ is assumed to be the same under the true and the subjective measures 5

Differences between investors' beliefs and the true model with respect to the parameters of the process for the conditionally expected growth rate $\mu$ have direct economic interpretations. For example, if investor $i$ assumes $\alpha_{s, i} \neq \alpha_{s}=0$, this is sometimes referred to as 'overconfidence' (see, e.g., Scheinkman and Xiong (2003) and Dumas et al. (2009) ), since she assigns more informational value to the signal

\footnotetext{
${ }^{4}$ The focus of our analysis is on the implications of these heterogeneous beliefs for survival and asset pricing. We thus take the beliefs of the investors as given and do not look at the reason why a certain investor type exhibits a certain type of error.

${ }^{5}$ A comparison with Dumas et al. (2009) shows that we have incorporated a potential correlation between the signal and the expected growth rate into the dynamics of $\mu$, while Dumas et al. (2009) assume that the two investors differ in their assumptions about the noise term in $d s_{t}$. In terms of the dynamics of the filtered estimate for $\mu$, these two formulations are equivalent.
} 
than it actually has. Analogously, the assumption $\alpha_{D, i} \neq \alpha_{D}=0$ means that investor $i$ considers the dividend to be more informative about the expected growth rate than it is under the true model. One could interpret $\alpha_{D, i}>0$ as some kind of momentum belief, while the opposite would represent a contrarian attitude, where in the investor's opinion higher dividends now would signal lower expected growth in the future. In our model there is thus a second potential source for overconfidence, since the investors can not only assign too much information value to the signal, but also to the dividend.

Assuming a value for the long-run mean of the expected growth rate $\bar{\mu}_{i} \neq \bar{\mu}$ represents (long-run) optimism or pessimism, while $\sigma_{\mu, i} \neq \sigma_{\mu}$ reflects investor $i$ 's subjective beliefs about the degree of uncertainty in $\mu_{t}$ or, technically speaking, about how well the expected growth rate can be filtered from the observable quantities. The case $\sigma_{\mu, 1}<\sigma_{\mu, 2}$ could therefore be interpreted as investor 1 being an 'expert', while investor 2 would be more cautious concerning the ability to filter information about $\mu$ from the observable data and thus be considered a 'layman'.

Finally, investors can also assume $\kappa_{i} \neq \kappa$, which is in a sense an assumption about the overall degree of uncertainty in $\mu$. A very high value for $\kappa_{i}$ means that investor $i$ assumes there is only little variation in $\mu$, especially over the long term, since the expected growth rate is always pulled back strongly towards its long-run mean $\bar{\mu}_{i}$. 


\subsection{Filtering}

We now turn to the formal representation of how investors form their expectations about the current value of $\mu$, given the data on $D$ and $s$. As stated above investors can differ with respect to their assumptions concerning the dynamics of the expected dividend growth rate $\mu$. Given the dynamics assumed by investor $i$ shown above in Equations (4) to (6) the standard theory of continuous-time filtering (see Liptser and Shiryaev (2001)), together with the dividend and signal dynamics from (1) and (2), implies the following investor-specific process $m_{i}(i=1,2)$ for the estimate of the unobservable expected growth rate:

$$
d m_{t, i}=\kappa_{i}\left(\bar{\mu}_{i}-m_{t, i}\right) d t+\frac{\gamma_{t, i}+\alpha_{D, i} \sigma_{D} \sigma_{\mu, i}}{\sigma_{D}} d \widehat{W}_{D, t, i}+\alpha_{s, i} \sigma_{\mu, i} d W_{s, t}
$$

where $\gamma_{t, i}$ denotes the mean squared error of the current estimate, i.e., $\gamma_{t, i} \equiv$ $E_{t}^{\mathbb{P}^{i}}\left[\left(\mu_{t}-m_{t, i}\right)^{2}\right] . \widehat{W}_{D, i}$ is the Wiener process driving the dividend innovations as perceived by investor $i$, given her current estimate of $\mu_{t}$ (see below).

Concerning the mean squared error $\gamma_{t, i}$, Liptser and Shiryaev (2001) show that it satisfies a Ricatti differential in time $t$. As usual in the literature (see, e.g., Dumas et al. (2009) or Buraschi et al. (2014)) we assume that it has already reached its stationary value $\gamma_{i}$ given by

$$
\gamma_{i}=\sigma_{D}^{2}\left[-\left(\kappa_{i}+\frac{\sigma_{\mu, i} \alpha_{D, i}}{\sigma_{D}}\right)+\sqrt{\left(\kappa_{i}+\frac{\sigma_{\mu, i} \alpha_{D, i}}{\sigma_{D}}\right)^{2}+\left(\frac{\sigma_{\mu, i} \alpha_{\mu, i}}{\sigma_{D}}\right)^{2}}\right],
$$

so that we can replace $\gamma_{t, i}$ by $\gamma_{i}$ in (7). For the following analysis, we define $\beta_{i}=\gamma_{i}+$ $\alpha_{D, i} \sigma_{D} \sigma_{\mu, i}$. The differences in beliefs between the two investors regarding the current 
value of the expected growth rate are labelled $g_{t}$, with $g_{t} \equiv m_{t, 1}-m_{t, 2}$. Furthermore, the investor-specific estimation error at time $t$ is denoted by $g_{t, i} \equiv m_{t, i}-\mu_{t}$.

The focus of our analysis is on the effects of different kinds of misspecification of the filtering process for investor survival and asset pricing. For these purposes the dynamics of the economy under the true probability measure $\mathbb{P}$ are central ${ }^{6}$

From Equation (1) for the dividend dynamics and the fact that the dividend is observable we get for $i=1,2$

$$
\begin{aligned}
\frac{d D_{t}}{D_{t}} & =\mu_{t} d t+\sigma_{D} d W_{D, t} \\
& \equiv m_{t, i} d t+\sigma_{D} d \widehat{W}_{D, t, i}
\end{aligned}
$$

which implies

$$
d \widehat{W}_{D, t, i}=d W_{D, t}+\frac{\mu_{t}-m_{t, i}}{\sigma_{D}} d t
$$

as well as

$$
d \widehat{W}_{D, t, 2}=d \widehat{W}_{D, t, 1}+\frac{g_{t}}{\sigma_{D}} d t
$$

From this we obtain the dynamics of the change of measure process $\eta \equiv \frac{d \mathbb{P}_{2}}{d \mathbb{P}_{1}}$ between the investors' subjective measures as

$$
d \eta_{t}=-\eta_{t} \frac{g_{t}}{\sigma_{D}} d \widehat{W}_{D, t, 1}
$$

\footnotetext{
${ }^{6}$ Note that to purely compute the price of the dividend claim and other traded assets we would not need to look at the true measure. However, we want to look at the asset pricing moments as seen by an outside observer or econometrician.
} 
Using Equations (5), (7), and (10) one obtains the following $\mathbb{P}$-dynamics for the true drift $\mu$, the investor-specific estimates $m_{i}$, and the change of measure $\eta$ :

$$
\begin{aligned}
d \mu_{t}= & \kappa\left(\bar{\mu}-\mu_{t}\right) d t+\sigma_{\mu}\left(\alpha_{D} d W_{D, t}+\alpha_{s} d W_{s, t}+\alpha_{\mu} d W_{\mu, t}\right) \\
d m_{t, i}= & \kappa_{i}\left(\bar{\mu}_{i}-m_{t, i}\right) d t+\frac{\beta_{i}}{\sigma_{D}^{2}}\left(\mu_{t}-m_{t, i}\right) d t \\
& +\frac{\beta_{i}}{\sigma_{D}} d W_{D, t}+\alpha_{s, i} \sigma_{\mu, i} d W_{s, t} \quad(i=1,2) \\
d \eta_{t}= & \eta_{t}\left[\frac{\left(m_{t, 1}-\mu_{t}\right)\left(m_{t, 1}-m_{t, 2}\right)}{\sigma_{D}^{2}} d t+\frac{m_{t, 2}-m_{t, 1}}{\sigma_{D}} d W_{D, t}\right] .
\end{aligned}
$$

\subsection{Investor Preferences and Consumption Sharing}

We assume that investors are equipped with identical CRRA preferences with coefficient of risk aversion $\nu \in \mathbb{N}]^{7}$ While in many applications more flexible preferences like recursive utility are used, CRRA utility still represents a benchmark case and allows direct comparison of our results to, e.g., Dumas et al. (2009). Formally, investor $i(i=1,2)$ derives utility from her consumption $C_{t, i}$ according to

$$
u\left(C_{t, i}\right)=\frac{C_{i, t}^{1-\nu}}{1-\nu}
$$

\footnotetext{
${ }^{7}$ Setting the degree of risk aversion equal to an integer has the advantage that the investor specific pricing kernel can be computed exactly due to the binomial formula. Bhamra and Uppal (2014) show that there is a series expansion which allows such a (semi-)closed form also for arbitrary coefficients of risk aversion. Since we do not estimate model parameters in this paper, we do not consider it a serious restriction to set risk aversion to an integer value. In our numerical analysis below we set $\nu=3$.
} 
so that the infinite-horizon lifetime utility is given by

$$
\int_{0}^{\infty} e^{-\delta t} \frac{C_{i, t}^{1-\nu}}{1-\nu} d t
$$

\subsection{Equilibrium}

The dynamic equilibrium in this economy is characterized by a consumption sharing rule $\omega_{t}$, which we represent as investor 2's share in aggregate consumption, i.e., $\omega_{t} \equiv \frac{C_{t, 2}}{C_{t}}$. We assume a complete market, and the investors have to agree on the prices of all traded assets 8 This gives some relation between the individual pricing kernels and the process $\eta$ for the change of measure from which we can then derive the consumption sharing rule. This consumption sharing rule is given as

$$
\omega_{t}=\frac{\left(\frac{\eta_{t}}{\phi_{2}}\right)^{\frac{1}{\nu}}}{\left(\frac{\eta_{t}}{\phi_{2}}\right)^{\frac{1}{\nu}}+\left(\frac{1}{\phi_{1}}\right)^{\frac{1}{\nu}}}
$$

where $\phi_{1}$ and $\phi_{2}$ are the Lagrange multipliers for the budget restrictions of investor 1 and 2, respectively, and where $\eta$ is the change of measure given in Equation (12).9

\footnotetext{
${ }^{8}$ Note that we deal with two filtrations in our model, a larger one generated by the signal, the dividend, and the true expected growth rate, and a smaller one generated by only the signal and the dividend. By assumption investors only observe events in the smaller filtration, and market completeness then of course refers to this smaller filtration, since claims on an unobservable quantity cannot be traded in a meaningful way. Stated differently, the existence of tradable claims on dividend and signal risk are sufficient for the market to be complete. For a discussion of this issue see also Basak (2005).

${ }^{9}$ The proof can be found in Appendix Appendix A.3
} 
The consumption sharing rule is the basis for all equilibrium results in the model. The pricing kernels of investor 1 and 2 are given by

$$
\begin{aligned}
& \xi_{t}^{1}=e^{-\delta t}\left(\frac{C_{t, 1}}{C_{0,1}}\right)^{-\nu}=e^{-\delta t}\left(\frac{\left(1-\omega_{t}\right) D_{t}}{\left(1-\omega_{0}\right) D_{0}}\right)^{-\nu} \\
& \xi_{t}^{2}=e^{-\delta t}\left(\frac{C_{t, 2}}{C_{0,2}}\right)^{-\nu}=e^{-\delta t}\left(\frac{\omega_{t} D_{t}}{\omega_{0} D_{0}}\right)^{-\nu} .
\end{aligned}
$$

The equilibrium stock price $S_{t}$ at time $t$ is equal to the present value of all future dividends paid at times $u \geq t$. Using investor $i$ 's pricing kernel leads to the following expression for $S_{t}$ :

$$
S_{t}=E_{t}^{\mathbb{P}^{i}}\left[\int_{t}^{\infty} e^{-\delta(u-t)} D_{u}\left(\frac{C_{u, i}}{C_{t, i}}\right)^{-\nu} d u\right]
$$

Since the investors agree on the stock price we can compute it under either $\mathbb{P}^{1}$ or $\mathbb{P}^{2}$, and we choose to take investor 1's perspective. A key ingredient to the computation of the stock price are thus the dynamics of the risk factors $D, \eta, m_{1}$, and $m_{2}$ under investor 1's measure. For the dividend $D$ and the drift estimate $m_{1}$ these are given for $i=1$ in Equations (7) and (9), respectively. The process for the change of measure $\eta$ is given in (12). For $m_{2}$ we obtain from (7) (for $i=2$ ) and 11)

$$
d m_{t, 2}=\left[\kappa_{2}\left(\bar{\mu}_{2}-m_{t, 2}\right)+\frac{\beta_{2}\left(m_{1}-m_{2}\right)}{\sigma_{D}^{2}}\right] d t+\frac{\beta_{2}}{\sigma_{D}} d \widehat{W}_{D, t, 1}+\alpha_{s, 2} \sigma_{\mu, 2} d W_{s, t}
$$

Given these dynamics, we can determine the joint characteristic function of $D$ and $\eta$. This allows us to calculate the present value of a single future dividend payment, and the stock price follows from taking the integral of these present values from the 
current point in time until infinity. ${ }^{10}$

Analogously to the stock price each investor's individual wealth is given by the present value of her consumption stream over time. Denoting investor $i$ 's wealth at time $t$ by $X_{t, i}$ this implies

$$
X_{t, i}=E_{t}^{\mathbb{P}^{i}}\left[\int_{t}^{\infty} e^{-\delta(u-t)} C_{u, i}\left(\frac{C_{u, i}}{C_{t, i}}\right)^{-\nu} d u\right]
$$

In the following, we are interested in the dynamics of the stock price and of wealth, i.e., in their volatilities and expected excess returns. Given the expression for the equilibrium stock price the local volatility vector $\sigma_{S} \equiv\left(\sigma_{S, D}, \sigma_{S, s}\right)$ of the return on equity can be computed as the product of the sensitivity matrix with respect to the risk factors $D, \eta, m_{1}$, and $m_{2}$, and the vector of volatilities of these factors with respect to the driving Wiener processes $\widehat{W}_{D, 1}$ and $W_{s}$ :

$$
\sigma_{S}=\frac{1}{S}\left(\frac{\partial S}{\partial D}, \frac{\partial S}{\partial \eta}, \frac{\partial S}{\partial m_{1}}, \frac{\partial S}{\partial m_{2}}\right)\left(\begin{array}{cc}
\sigma_{D} D & 0 \\
\frac{\eta_{t}\left(m_{t, 2}-m_{t, 1}\right)}{\sigma_{D}} & 0 \\
\beta_{1} / \sigma_{D} & \alpha_{s, 1} \sigma_{\mu, 1} \\
\beta_{2} / \sigma_{D} & \alpha_{s, 2} \sigma_{\mu, 2}
\end{array}\right) .
$$

The overall return volatility $\sigma_{R}$ of the stock is given by the norm of $\sigma_{S}$, i.e., it is equal to $\sqrt{\sigma_{S} \sigma_{S}^{\prime}}$.

Finally, the expected excess return is given by the product of the vector $\sigma_{S}$ and the vector of market prices of risk for the two driving Wiener processes 11 Note

\footnotetext{
${ }^{10}$ The details of the computation of the stock price are given in Appendix Appendix A.1.

${ }^{11}$ The market prices of risk are derived in Appendix Appendix A.5.
} 
that the market prices of risk can be computed both under the investors' subjective measures $\mathbb{P}^{i}$ and under the objective measure $\mathbb{P}$. We are interested in the properties of expected excess stock returns as they would be observed by an econometrician analyzing the data generated by our model, i.e., we select the objective measure as our perspective here.

Again in analogy to the stock price we can compute the volatility vector $\sigma_{X_{i}}$ of investor $i$ 's wealth as

$$
\sigma_{X_{i}}=\frac{1}{X_{i}}\left(\frac{\partial X_{i}}{\partial D}, \frac{\partial X_{i}}{\partial \eta}, \frac{\partial X_{i}}{\partial m_{1}}, \frac{\partial X_{i}}{\partial m_{2}}\right)\left(\begin{array}{cc}
\sigma_{D} D & 0 \\
\frac{\eta_{t}\left(m_{t, 2}-m_{t, 1}\right)}{\sigma_{D}} & 0 \\
\beta_{1} / \sigma_{D} & \alpha_{s, 1} \sigma_{\mu, 1} \\
\beta_{2} / \sigma_{D} & \alpha_{s, 2} \sigma_{\mu, 2}
\end{array}\right) .
$$

The overall volatility of the return on investor $i$ 's wealth is given by $\sqrt{\sigma_{X_{i}} \sigma_{X_{i}}^{\prime}}$, and the expected excess return on each investor's wealth under the objective measure $\mathbb{P}$ is obtained by multiplying the volatility vector by the associated market prices of risk.

An important aspect of a certain parameter combination is whether the model is meaningful in the sense that it generates a finite stock price. Under investor $i$ 's subjective measure the growth condition derived in Appendix Appendix A.2 is given by the inequality

$$
(1-\nu)\left(\bar{\mu}_{i}-\frac{1}{2} \sigma_{D}^{2}\right)+\frac{(1-\nu)^{2}}{2}\left[\sigma_{D}^{2}+\left(\frac{\beta_{i}}{\sigma_{D} \kappa_{i}}\right)^{2}+\left(\frac{\alpha_{s, i} \sigma_{\mu, i}}{\kappa_{i}}\right)^{2}+\frac{2 \beta_{i}}{\kappa_{i}}\right]<\delta
$$


It basically states that the subjective discount rate $\delta$ must be large enough so that the present value of future payments goes to zero when the horizon goes to infinity. Since both investors assume that they will survive under their respective subjective model, the growth condition has to hold under both $\mathbb{P}^{1}$ and $\mathbb{P}^{2}{ }^{12}$

\subsection{Survival}

The investors make consumption and investment choices according to their preferences and their current estimate of the growth rate $\mu$. In case only one of the two investors uses the correct model, while the other one makes improper assumptions about the dynamics of the expected growth rate, it is intuitively clear that the investor with the incorrect model will be at a disadvantage in terms of her utility. In the long run she will even loose all of her consumption, so that the economy will become homogeneous again. In our case we have to track how the investors' consumption shares evolve over time to see which of the two types will eventually survive.

Borovicka (2015) provides a formal representation of investor survival, and we follow his terminology here ${ }^{13}$ Consider a path $\bar{\omega}$ of the economy which is characterized by the realizations of the driving Wiener processes $W_{D}, W_{s}$, and $W_{\mu}$ over time. We say that investor 1 becomes extinct along this path, if the limit for $t \rightarrow \infty$ of her

\footnotetext{
${ }^{12}$ In the quantitative analysis of the model in Section 3 , we restrict the discussion to parameter combinations where the growth conditions are satisfied.

${ }^{13}$ In particular, see Definition 4 on p. 17 of his paper.
} 
consumption share goes to zero, i.e., if $\lim _{t \rightarrow \infty} \omega_{1, t}(\bar{\omega})=0$. If this is not the case, then investor 1 is said to survive along the path. Furthermore, investor 1 is said to dominate along the path, if her consumption share goes to 1, i.e., if $\lim _{t \rightarrow \infty} \omega_{1, t}(\bar{\omega})=1$. We later on focus on the probability of an investor surviving or not. More precisely we say that investor 1 becomes extinct under the measure $\mathbb{P}$, if $\mathbb{P}\left(\lim _{t \rightarrow \infty} \omega_{1, t}(\bar{\omega})=0\right)=1$, and accordingly, the investor survives under $\mathbb{P}$, if $\mathbb{P}\left(\lim \sup _{t \rightarrow \infty} \omega_{1, t}(\bar{\omega})>0\right)=1$. Finally, she is said to dominate under $\mathbb{P}$, if $\mathbb{P}\left(\lim _{t \rightarrow \infty} \omega_{1, t}(\bar{\omega})=1\right)=1$.

As discussed in Borovicka (2015) the probabilistic statements about survival can be made operational via the properties of the drift of an investor's consumption share, when she becomes small, i.e., when her consumption share tends to zero. More precisely, an investor will vanish when the drift of her (log) consumption share is negative in the limit for the consumption share going to zero.

In our model there is a state variable, and this fact makes things much more complicated. The drift of the consumption share in general depends on these state variables, and one thus needs the joint distribution of this drift and the state variable in the long run to analyze survival. To get the intuition, assume that the drift of the consumption share $\omega$ for a small $\omega$ can be positive or negative, depending on the state variable. Now consider the following two simplified cases. In the first case, a small consumption share can only occur for those values of the state variable for which the drift of $\ln \omega$ is positive. In the second case, a small consumption share can only occur for those values of the state variable for which the drift of $\ln \omega$ is negative. 
While the investor survives in the first case, she becomes extinct in the second one. It is thus the joint distribution of the consumption share and the state variable which matters for survival. While the expression for the drift of the consumption share as $\omega$ tends to zero and the long-run distribution of $\mu$ are relatively easy to obtain individually, finding their joint distribution is far from straightforward.

To analyze survival we thus rely on the evolution of consumption share densities over time, as it is commonly done in the literature (see, e.g., Dumas et al. (2009) or Easley and Yang (2013)). This has the additional advantage that we also get an idea of the speed of 'natural selection' in the market. The most important statistic in this context will be the investors' expected consumption shares at future points in time.

To compute the density of the consumption share and then its expectation for some future point in time $u$ one first has to find the characteristic function for the change of measure $\eta_{u}$, since as shown above in (16), the consumption share is a function of $\eta$. We thus first compute $H^{\mathbb{P}}\left(m_{1}, m_{2}, \mu, \eta, t, u ; \chi\right) \equiv E_{t}^{\mathbb{P}}\left[\eta_{u}^{\chi}\right]$, which is assumed to have an exponentially affine-quadratic form, i.e.,

$$
\begin{aligned}
H^{\mathbb{P}}\left(m_{1}, m_{2}, \mu, \eta, t, u ; \chi\right) \equiv & \eta^{\chi} \bar{H}^{\mathbb{P}}\left(m_{1}, m_{2}, \mu, \chi ; \tau\right) \\
= & \eta^{\chi} \exp \left(A+B_{1} m_{1}+B_{2} m_{2}+B_{3} \mu+C_{11} m_{1}^{2}+2 C_{12} m_{1} m_{2}\right. \\
& \left.+2 C_{13} m_{1} \mu+C_{22} m_{2}^{2}+2 C_{23} m_{2} \mu+C_{33} \mu^{2}\right)
\end{aligned}
$$

with $\tau \equiv u-t$. The functions $A, \ldots, C_{33}$ all depend on $\tau$ and $\chi$ and satisfy ordinary 
differential equations derived in Appendix Appendix A.6. The initial condition is the same for all functions, namely that the function value for $\tau=0$ is equal to zero. Given the function $H^{\mathbb{P}}\left(m_{1}, m_{2}, \mu, \eta, t, u ; \chi\right)$ the density as of time $t$ of the change of measure at time $u$ is obtained as

$$
f\left(\eta_{u} ; m_{1, t}, m_{2, t}, \mu_{t}, \eta_{t}, t, u\right)=\frac{1}{2 \pi} \int_{-\infty}^{\infty} \eta_{u}^{-i \chi} H^{\mathbb{P}}\left(m_{1, t}, m_{2, t}, \mu_{t}, \eta_{t}, t, u ; i \chi\right) \frac{1}{\eta_{u}} d \chi
$$

and the expectation of the consumption share $\omega$ as a function of $\eta$ can be computed as

$$
E_{t}^{\mathbb{P}}\left(\omega_{u}\right)=\int_{0}^{\infty} \omega\left(\eta_{u}\right) f\left(\eta_{u} ; m_{1, t}, m_{2, t}, \mu_{t}, \eta_{t}, t, u\right) d \eta_{u} .
$$

The behavior of this expected consumption share for different values of $\tau$ will serve as our measure for survival in models where both investors exhibit imperfect filtering models.

\section{Quantitative Analysis of the Model}

\subsection{Survival}

Our goal is to analyze situations where both investors suffer from an imperfect learning mechanism, and the question is whether or not investor 1's 'mistake' will be compensated by investor 2's problem such that investor 1 will survive in the long run. We consider situations where an investor assigns too much informational value to the signal or the dividend by assuming $\alpha_{s, i} \neq 0$ or $\alpha_{D, i} \neq 0$ (labeled 'signal overconfidence' and 'dividend overconfidence', respectively), where she exhibits what 
we call a 'long-term growth bias' by assuming $\bar{\mu}_{i} \neq \bar{\mu}$, where she assumes an incorrect value for the volatility of the expected growth rate process $\left(\sigma_{\mu, i} \neq \sigma_{\mu}\right)$, implying that she believes the expected growth rate can be more or less precisely estimated than under the true model, or where she assumes $\kappa_{i} \neq \kappa$ and thus has a different belief about the overall variation in the expected growth rate. The parameters under the objective measure $\mathbb{P}$ are given in Table A.1.

We look at the expectation of investor 2's consumption share $\omega$ for horizons of $\tau=50,200$, and 500 years and take the evolution of this variable as an indication of survival of only one or both of the investors. We present the output in the form of contour plots, where the contour lines indicate parameter combinations, which all yield one given expected consumption share for investor 2. So values in the contour plot below 0.5 indicate parameter combinations where investor 2 looses expected consumption over time and vice versa. The knife-edge case, where both investors survive in the long run, is represented by the contour lines representing a value for the expectation of $\omega$ equal to 0.5 .

We will now discuss a number of different scenarios concerning the investors' filtering errors and analyze the implications for survival. Given our general setup, we could discuss any pair of misspecified models ${ }^{14}$ but we chose to restrict the analysis

\footnotetext{
${ }^{14}$ Actually we could also analyze cases when both investors commit errors with respect to more than one parameter in the model. For reasons of parsimony, however, we opted for the 'cleaner' setup with only one type of error for each investor.
} 
to the cases when one of the investors is overconfident, be it with respect to the signal $\left(\alpha_{s, i} \neq 0\right)$ or with respect to the dividend $\left(\alpha_{D, i} \neq 0\right)$. All the analyses are restricted to the parameter combinations which yield a finite stock price, i.e., which satisfy the growth condition for the stock price.

Figure A.1 shows contour plots of investor 2's expected consumption share $\omega$, when investor 2 is overconfident with respect to the signal, and investor 1 uses an incorrect volatility of expected growth in her filtering model. The situation discussed in Dumas et al. (2009) with one overconfident and one rational investor, i.e., with $\alpha_{s, 2}=0.95$ and $\sigma_{\mu, 1}=\sigma_{\mu}=0.03$, is one special case of the parameter combinations analyzed here.

The figure first confirms that in the case where only one investor makes a mistake the consumption share of this investor will decrease over time. Much more important, however, it allows us to analyze the trade-off between the two types of errors considered here. Intuitively, it is clear that the more $\alpha_{s, 2}$ deviates from the true value of zero, the more $\sigma_{\mu, 1}$ has to deviate from the true value of 0.03 for investor 2's expected consumption share to remain at or above 0.5 . The contour lines for $\omega=0.5$ give the combinations of parameters for the knife-edge case in which both investors survive.

One might expect that there is always a threshold for the other investor's model misspecification which allows investor 2 to survive. The key conclusion that we can draw from the graphs in Figure A.1 is, however, that a too high degree 
of investor 2's overconfidence (beyond approximately 0.5 in absolute value for our parameterization), can no longer be compensated by a deviation of $\sigma_{\mu, 1}$ from its true value. The stock price growth condition imposes an upper bound of approximately 0.04 on $\sigma_{\mu, 1}$, which means that there are no admissible models with a higher value for this parameter. This implies that investor 2 will finally face a zero expected consumption share when she uses a model with $\alpha_{s, 2}$ too large in absolute value. Finally, the contour plot is symmetric with respect to the line represented by $\alpha_{s, 2}=$ 0 , so that the sign of $\alpha_{s, 2}$ does not matter.

Switching perspective, the question is of course also whether a too high deviation of $\sigma_{\mu, 1}$ from the true value $\sigma_{\mu}$ can be compensated by a sufficiently large signal overconfidence of investor 2. Figure A.1 shows that this is indeed the case for all values of $\sigma_{\mu, 1}$ for which the growth condition is met. We can always find a value of $\alpha_{s, 2}$ for which the expected consumption share of investor 2 drops below 0.5 and the expected consumption share of investor 1 is thus above 0.5 . In terms of survival, signal overconfidence is thus a worse model misspecification than being an expert $\left(\sigma_{\mu_{1}}<\sigma_{\mu}\right)$ or a laymen $\left(\sigma_{\mu_{1}}>\sigma_{\mu}\right)$.

Figure A.2 analyzes the trade-off between signal overconfidence and optimism or pessimism concerning the long-run expected growth rate of dividends. Again, signal overconfidence of investor 2 is the much more severe mistake. The area where investor 2 retains an expected consumption share of 50 percent or more over the long term is rather narrow and the slope of the line separating the two survival regions 
is rather flat in the direction of $\bar{\mu}_{1}$. Even if investor 1 also commits an error by being overly optimistic or pessimistic, this error has to be substantial to let investor 2 survive. For example, an absolute value of $\alpha_{s, 2}$ greater than around 0.25 means that investor 1 would have to assume a value of $\bar{\mu}_{1}$ outside the range shown in our graphs for investor 2 to survive, i.e., either assume a negative long-run mean of the expected growth rate or a value more than twice the true one.

Nevertheless, the important difference of this case compared to the situation when investor 1 had misspecified $\sigma_{\mu}$ is that there would (at least in principle) always be a value of $\bar{\mu}_{1}$ that would give investor 2 an expected consumption share of 0.5 or greater by plugging in values for $\bar{\mu}_{1}$ to the left and the right of the interval shown in the figure.

Next, Figure A.3 compares signal overconfidence of investor 2 to incorrect assumptions of investor 1 about the mean-reversion speed $\kappa_{1}$. The growth condition now implies a lower bound on the viable values of $\kappa_{1}$. The structural form of the contour lines is very similar to the one for the previous case when $\bar{\mu}_{1}$ was not equal to the true value. In particular, the contour lines in the direction of $\kappa_{1}$ are rather flat. It thus needs a high value of $\kappa_{1}$ to compensate a given value $\alpha_{s, 2}$ of signal overconfidence.

It is also of interest to compare the two forms of overconfidence in our model 
directly. Figure A.4 shows the contour plots for the case $\alpha_{D, 1} \neq 0$ and $\alpha_{s, 2} \neq 015$ The results confirm that signal overconfidence is a severe mistake even compared to dividend overconfidence. If the absolute values of $\alpha_{D, 1}$ and $\alpha_{s, 2}$ coincide, the investors assume the same overall level of correlation between (unobservable) innovations in the expected growth rate and either dividend innovations or signal innovations. Despite the apparently same amount of model misspecification, the figure shows that it will always be investor 2 who is driven out of the market in the long run, i.e., the investor who is overconfident with respect to the information content of the signal.

Finally, Figure A.5 gives the contour plot for dividend overconfidence on the part of investor $2\left(\alpha_{D_{2}} \neq 0\right)$ and investor 1 being a 'laymen' or an 'expert' $\left(\sigma_{\mu_{1}} \neq \sigma_{\mu}\right)$. A comparison with Figure A.1 allows to assess the difference between the case analyzed here and the case of signal overconfidence presented in Figure A.1. Analogous to the case of signal overconfidence there is a critical value for $\alpha_{D, 2}$ (here it is around $-0.55)$, below which there is no $\sigma_{\mu, 1}$ for which investor 2 would survive in the long run. Unlike in the first case, however, where the contour lines for an expectation of $\omega$ equal to 0.5 are very close to being symmetric around the line given by $\alpha_{s, 2}=0$

\footnotetext{
${ }^{15}$ In our numerical analysis we restrict $\alpha_{D}$ to be greater than or equal to -0.5 (see Table A.1). The reason for this restriction is that positive dividend innovations per se always induce an upward revision in the estimate for the expected growth rate, while a negative value for $\alpha_{D}$ implies just the opposite, and we want to exclude cases where this opposing force becomes too strong.
} 
in Figure A.1, survival is no longer symmetric around the true model with $\alpha_{D}=0$. Being erroneously 'contrarian' with $\alpha_{D, 1}<0$, i.e., believing in a model where higher dividend innovations today signal lower expected growth rates in the future, seems much worse than believing in 'momentum', i.e., using a model with $\alpha_{D, 1}>0$. For example, when $\alpha_{D, 2}=0.4$, investor 2 will survive for any value of $\sigma_{\mu, 1}$ less than approximately 0.02 , while for $\alpha_{D, 2}=-0.4$ this will only happen when investor 1 assumes a volatility of the expected growth rate process below roughly 0.016 .

Furthermore, the slope of the lines for the knife edge case are now much steeper in $\sigma_{\mu, 1}$-direction than in the case of signal overconfidence. This implies that it is actually a more severe problem for investor 1 to misspecify the volatility of the expected growth rate process when investor 2 is overconfident with respect to the dividend than when she assigns too much information value to the signal. As we can see in the graphs in Figure A.5 moving away from the true value of $\sigma_{\mu, 1}=0.03$ means that investor 2 can misspecify $\alpha_{D, 2}$ by more than she was 'allowed' to misspecify $\alpha_{s, 2}$ to retain an expected consumption share of at least 0.5 . In positive $\alpha_{D, 2^{2}}$-direction the line is even steeper, in line with the previously described asymmetry. One reason for dividend overconfidence being less severe a mistake than signal overconfidence can be seen in the fact that dividend innovations are per se informative about the expected growth rate, since Equation (7) shows that even with $\alpha_{D, i}=\alpha_{s, i}=0$ the perceived dividend innovation has a positive impact on the change in $m$, in contrast to the signal, which in itself does not contain any information under the 
true model. An incorrect value of $\alpha_{s, 2}$ thus implies that signal innovations are in a sense overweighted in a more pronounced fashion than dividend innovations are for an equally large value of $\alpha_{D, 2}$.

\subsection{Asset Pricing Implications}

In terms of the asset pricing implications of the model we are interested in volatilities and expected excess returns for both the aggregate stock market and individual investor wealth. The expressions for the volatilities of the stock return and the return on the individual wealth levels are given in Equations $(19)$ and $(20)$. The expected excess returns are obtained by multiplying these volatilities by the market prices of risk shown in Appendix Appendix A.5.

The original effect of introducing an overconfident investor into the standard

model as in, e.g., Dumas et al. (2009) was to increase the expected equity return and the volatility of stock returns which also brought the model closer to empirically observed values. The more fundamental question in our model now is how the introduction of a second imperfect investor impacts the asset pricing moments. Starting from the point where only one investor has an imperfect learning model the fact that also the second investor commits an error makes the two investors in a sense more similar. As we had seen in the previous section, there is indeed a trade-off between various mistakes when it comes to survival, and the question is whether this is also true for asset pricing. While these arguments point towards a smaller 
impact on asset prices when nobody is perfect than when only one investor uses an incorrect learning model, the additional mistake of the second investor makes the process of price formation in a sense deviate even further from the process if both investors would be right. This shows that one ultimately has to compute expected excess returns and volatilities explicitly to see the overall effect of the setup where 'nobody is perfect'.

We go through the scenarios in the same sequence as in the section on survival. Figure A.6 shows the results for $\alpha_{s, 2}$ and $\sigma_{\mu, 1}$ being different from the respective true values. Both volatilities and expected excess returns are increasing in the absolute value of $\alpha_{s, 2}$, i.e., in the signal overconfidence of investor 2. They are decreasing in $\sigma_{\mu, 1}$ for small and medium values of $\sigma_{\mu, 1}$ and can start to increase again for high values of $\sigma_{\mu, 1}$, but stay below the volatility for $\sigma_{\mu, 1}=\sigma_{\mu}$. Stated differently, volatilities and expected excess returns are the larger the more investor 1 considers herself an expert.

The striking observation, however, is that asset pricing moments behave quite differently than expected consumption shares. In particular, when it comes to asset pricing, there is nothing special about those parameter combinations for which both investors maintain an average consumption share of 0.5 and are thus most similar in terms of survival. As an example, take the situation where $\sigma_{\mu, 1}$ is rather low and look at the expected excess return on the stock for varying $\alpha_{s, 2}$. Now going from low to high values for this parameter we see that the volatilities of stock returns 
and both investors' individual wealth levels first become smaller, before they reach a minimum around $\alpha_{s, 2}=0$ and then start to increase again. In terms of expected consumption shares and survival (see Figure A.1), however, we see starting from low values of $\alpha_{s, 2}$ the investors first become more similar with respect to expected consumption shares, since the first knife edge case is reached for $\alpha_{s, 2}$ around -0.45 . Increasing $\alpha_{s, 2}$ further beyond this point makes investors more different in terms of survival, but volatilities still decrease until $\alpha_{s, 2} \approx 0$. The situation for positive $\alpha_{s, 2}$ is basically symmetric, but with opposite signs.

Figure A.7, A.8, and A.9 present the results for the cases where investor 2 is still overconfident concerning the signal, while investor 1 makes a mistake concerning the average expected growth rate, the mean-reversion speed of the expected growth rate, or is overconfident concerning the dividend. In all cases, volatilities and expected excess returns are basically a U-shaped function of $\alpha_{s, 2}$ for any given value of investor 1's parameter.

There are differences between the scenarios, however, concerning the shape of the return volatility surfaces for a given value of $\alpha_{s, 2}$, going in the direction of increasing values for investor 1's respective parameter. The volatilities stay more or less constant in $\bar{\mu}_{1}$ (Figure A.7), while they strongly increase in $\kappa_{1}$ (Figure A.8) and decrease in $\alpha_{D, 1}$ (Figure A.9). A comparison with the behavior of expected consumption shares again emphasizes that knowing the properties of the model with respect to the investors' consumption shares does not allow a conclusion concerning 
its asset pricing characteristics. While Figure A.3 showed that the value of $\kappa_{1}$ hardly played a role for the expected distribution of aggregate consumption across the two investors, $\kappa_{1}$ has a pronounced impact on return volatilities. They range from values as low as 0.02 for low values of $\kappa_{1}$ and $\alpha_{s, 2}$ around zero to approximately 0.06 for high values of $\kappa_{1}$. On the other hand return volatilities are hardly sensitive to $\bar{\mu}_{1}$, just like expected consumption shares, given a certain value for $\alpha_{s, 2}$. Finally, both expected consumption shares and volatilities are sensitive to dividend overconfidence $\alpha_{D, 1}$, with the impact on volatilities being larger than the impact on expected consumption shares. The effects for expected excess returns are very similar to those for volatilities, since the risk premium is obtained by simply multiplying volatilities by the market price of risk under the true measure.

Again, a comparison of signal overconfidence and dividend overconfidence is of interest to assess how much it matters for asset pricing moments what the source of an investor's overconfidence actually is. We first consider the case where investor 1 considers herself an expert $\left(\sigma_{\mu, 1}<\sigma_{\mu}\right)$ or a layman $\left(\sigma_{\mu_{1}}>\sigma_{\mu}\right)$, while investor 2 is overconfident with respect to the signal (Figure A.6) or the dividend (Figure A.10). The implications for asset pricing moments are very different. While volatilities are increasing in the absolute value of signal overconfidence $\alpha_{s, 2}$, they are a decreasing function of dividend overconfidence $\alpha_{D, 2}$.

In the next step we compare the implications of signal overconfidence and dividend overconfidence directly. Figure A.9 shows volatilities and expected excess 
returns for the case $\alpha_{s, 2} \neq 0$ and $\alpha_{D, 1} \neq 0$. Again, volatilities are increasing in the absolute value of signal overconfidence, while they are decreasing in dividend overconfidence. Furthermore, the impact of $\alpha_{D, 1}$ on volatilities is larger than the impact of an equally large $\alpha_{s, 1}$. The correlations between expected consumption growth and dividends on volatilities is thus larger than the impact of its correlation with signals. Overall we thus see that it makes a big difference in terms of the model output whether the investor assigns too much informational value to the dividend or to a completely exogenous signal.

The most important conclusion from the analysis here is thus that the fact that investors are more similar in terms of survival or that even both survive (in the knife edge case) implies basically nothing about volatilities and expected returns. In this sense short-term asset pricing results are 'independent' of the long-term properties of the model.

\section{Conclusion}

In this paper we have analyzed an economy with heterogeneous investors. They differ in their assumptions on the true dynamics of the (unobservable) expected dividend growth rate and thus use different estimates which result from filtering this expected growth rate from observable data. The special feature of our model compared to the rest of the literature is that actually both investors use models which do no coincide with the true one. We propose a rather general setup in which 
the investors can disagree about any parameter of the latent process for the expected growth rate and analyze the implications of this 'nobody-is-perfect' setup for survival and asset pricing.

While it is clear that in terms of survival the disadvantage of the first investor with an imperfect learning model decreases when also the second investor commits an error, it is not clear what the trade-off between different mistakes is. E.g., is it more severe to use a misspecified correlation between the signal and the dividend than to use an improper volatility of the expected growth rate process? We investigate a number of different combinations of the types of errors committed by the two investors. One of the most striking results is that there are errors on the part of one investor which cannot be compensated by the other investor's deficiency. More precisely, with one investor's signal overconfidence exceeding a certain threshold, there is no degree of misspecification in the volatility of the expected growth rate process which could offset this disadvantage. We can conclude from this that in a certain sense over-confidence is a more severe problem than other forms of misspecified filtering models.

Investor survival represents a very long-term view on a model. It is also of interest how the presence of heterogeneous investors with different kinds of misspecified filtering models affects the asset pricing outcomes in terms of expected returns and volatilities of the aggregate stock market and individual investor wealth. The main finding here is that the two dimensions of the model, asset pricing and survival, 
are in a sense independent. In scenarios when the investors are more similar with respect to their expected consumption shares volatilities can nevertheless be higher than in cases when investors are very different, i.e., when the expected consumption shares deviate significantly from an equal distribution across investors. Asset pricing moments are by no means monotonous in investor similarity with respect to long run survival, and the implications of a model for asset pricing moments and survival basically have to be analyzed separately from each other. 


\section{References}

Basak, S., 2005. Asset pricing with heterogeneous beliefs. Journal of Banking and Finance 29, 2849-2881.

Bhamra, H.S., Uppal, R., 2014. Asset prices with heterogeneity in preferences and beliefs. Review of Financial Studies 27, 519-580.

Borovicka, J., 2015. Survival and long-run dynamics with heterogeneous beliefs under recursive preferences. Working Paper.

Buraschi, A., Trojani, F., Vedolin, A., 2014. When uncertainty blows in the orchard: Comovement and equilibrium volatility risk premia. Journal of Finance 69, 101137.

Dumas, B., Kurshev, A., Uppal, R., 2009. Equilibrium portfolio strategies in the presence of sentiment risk and excess volatility. Journal of Finance 64, 579-629.

Easley, D., Yang, L., 2013. Loss aversion, survival and asset prices. Working Paper.

Kogan, L., Ross, S., Wang, J., Westerfield, M., 2006. The price impact and survival of irrational traders. Journal of Finance 61, 195-229.

Liptser, R., Shiryaev, A.N., 2001. Statistics of Random Processes II. Applications. Springer Verlag, Berlin.

Scheinkman, J., Xiong, W., 2003. Overconfidence and speculative bubbles. Journal of Political Economy 111, 1183-1219. 
Yan, H., 2008. Natural selection in financial markets: Does it work? Management Science 54, 1935-1950. 


\section{Appendix A. Appendix}

Appendix A.1. Equilibrium Stock Price

Since the investors agree on the stock price, we are free to choose under which measure we want to compute the expectation of future discounted dividends, and we adopt investor 1's perspective. The key element in the expression for the stock price is the present value of a single future dividend payment, i.e., the expression

$$
E_{t}^{1}\left[e^{-\beta(u-t)}\left(\frac{C_{u, 1}}{C_{t, 1}}\right)^{\nu-1} D_{u}\right]=E_{t}^{1}\left[e^{-\beta(u-t)}\left(\frac{\left(1-\omega_{u}\right) D_{u}}{\left(1-\omega_{t}\right) D_{t}}\right)^{\nu-1} D_{u}\right] .
$$

Since the consumption sharing rule depends on the change of measure as shown in Equation (16), we need the joint moment generating function of future dividends and future measure changes. Let this function be represented as

$$
\begin{aligned}
E_{t}^{1}\left[D_{u}^{\epsilon} \eta_{u}^{\chi}\right] & =D_{t}^{\epsilon} \cdot \eta_{t}^{\chi} \cdot H\left(\epsilon, \chi ; t, u, m_{t, 1}, m_{t, 2}\right) \\
& \equiv D_{t}^{\epsilon} \cdot \eta_{t}^{\chi} \cdot H^{m}\left(\epsilon ; t, u, m_{1, t}\right) \cdot H^{g}\left(\epsilon, \chi ; t, u, m_{t, 1}, m_{t, 2}\right)
\end{aligned}
$$

with $H^{m}(\cdot)$ as the moment generating function for future dividends $s^{16}$, i.e.,

$$
D_{t}^{\epsilon} H^{m}\left(\epsilon ; t, u, m_{1, t}\right)=E_{t}^{1}\left[D_{u}^{\epsilon}\right]
$$

One can find $H^{m}$ by direct integration. Under investor 1's subjective probability measure her own filtered estimate for the expected growth rate and the dividend evolve according to

$$
\begin{aligned}
\frac{d D_{t}}{D_{t}} & =m_{t, 1} d t+\sigma_{D} d \widehat{W}_{D, t, 1} \\
d m_{t, 1} & =\kappa_{1}\left(\bar{\mu}_{1}-m_{t, 1}\right) d t+\frac{\beta_{1}}{\sigma_{D}} d \widehat{W}_{D, t, 1}+\alpha_{s, 1} \sigma_{\mu, 1} d W_{s, t}
\end{aligned}
$$

\footnotetext{
${ }^{16} H^{m}(\cdot)$ and $H^{g}(\cdot)$ are of course functions of all the parameters governing the subjective evolution of $m_{1}$ and the dividend process. However, to keep notation to a minimum we will most of the time suppress all the arguments.
} 
This implies

$$
\ln D_{u}=\ln D_{t}+\int_{t}^{u}\left(m_{v, 1}-\frac{1}{2} \sigma_{D}^{2}\right) d v+\sigma_{D} \int_{t}^{u} d \widehat{W}_{D, v, 1} .
$$

and

$m_{v, 1}=e^{-\kappa_{1}(v-t)}\left(m_{t, 1}-\bar{\mu}_{1}\right)+\bar{\mu}_{1}+\frac{\beta_{1}}{\sigma_{D}} \int_{t}^{v} e^{-\kappa_{1}(v-u)} d \widehat{W}_{D, u, 1}+\alpha_{s, 1} \sigma_{\mu, 1} \int_{t}^{v} e^{-\kappa_{1}(v-u)} d W_{s, u}$.

From this we obtain, using Fubini's theorem,

$$
\begin{aligned}
\int_{t}^{u} m_{v, 1} d v= & \frac{1}{\kappa_{1}}\left(1-e^{-\kappa_{1}(u-t)}\right)\left(m_{t, 1}-\bar{\mu}_{1}\right)+\bar{\mu}_{1}(u-t) \\
& +\frac{\beta_{1}}{\sigma_{D}} \int_{t}^{u} \frac{1}{\kappa_{1}}\left(1-e^{-\kappa_{1}(u-v)}\right) d \widehat{W}_{D, v, 1}+\alpha_{s, 1} \sigma_{\mu, 1} \int_{t}^{u} \frac{1}{\kappa_{1}}\left(1-e^{-\kappa_{1}(u-v)}\right) d \widehat{W}_{s, v} .
\end{aligned}
$$

This yields

$$
E_{t}^{\mathbb{P}^{1}}\left[\ln D_{u}\right]=\ln D_{t}+\frac{1}{\kappa_{1}}\left(1-e^{-\kappa_{1}(u-t)}\right)\left(m_{t, 1}-\bar{\mu}_{i}\right)+\bar{\mu}_{1}(u-t)-\frac{1}{2} \sigma_{D}^{2}(u-t)
$$

and

$$
\begin{aligned}
\operatorname{var}_{t}^{\mathbb{P}^{1}}\left[\ln D_{u}\right]= & \sigma_{D}^{2}(u-t)+\left[\left(\frac{\beta_{1}}{\sigma_{D}}\right)^{2}+\left(\alpha_{s, 1} \sigma_{\mu, 1}\right)^{2}\right] \int_{t}^{u}\left[\frac{1}{\kappa_{1}}\left(1-e^{-\kappa_{1}(u-v)}\right)\right]^{2} d v \\
& +2 \beta_{1} \int_{t}^{u}\left[\frac{1}{\kappa_{1}}\left(1-e^{-\kappa_{1}(u-v)}\right)\right] d v \\
= & \sigma_{D}^{2}(u-t) \\
& +\left[\left(\frac{\beta_{1}}{\sigma_{D} \kappa_{1}}\right)^{2}+\left(\frac{\alpha_{s, 1} \sigma_{\mu, 1}}{\kappa_{1}}\right)^{2}\right]\left[(u-t)-\frac{2\left(1-e^{-\kappa_{1}(u-t)}\right)}{\kappa_{1}}+\frac{\left(1-e^{-2 \kappa_{1}(u-t)}\right)}{2 \kappa_{1}}\right] \\
& +\frac{2 \beta_{1}}{\kappa_{1}}\left[(u-t)-\frac{1-e^{-\kappa_{1}(u-t)}}{\kappa_{1}}\right]
\end{aligned}
$$

Due to the lognormality of $D_{u}$ we finally obtain

$$
E_{t}^{1}\left[D_{u}^{\epsilon}\right]=\left(\epsilon E_{t}^{1}\left[\ln D_{u}\right]+\frac{1}{2} \epsilon^{2} \operatorname{var}_{t}^{1}\left[\ln D_{u}\right]\right) .
$$

We make the usual exponentially affine-quadratic guess for the function $H^{g}$ :

$$
H^{g}\left(m_{1}, m_{2}, \chi ; t, u\right) \equiv \exp \left(K+L_{1} m_{1}+L_{2} m_{2}+M_{11} m_{1}^{2}+2 M_{12} m_{1} m_{2}+M_{22} m_{2}^{2}\right),
$$


where $K, \ldots, M_{22}$ are functions of $\tau \equiv u-t, \epsilon$, and $\chi$ with boundary conditions $K(0)=L_{1}(0)=\ldots=M_{22}(0)=0$.

Note that, under investor 1's subjective measure, investor 2's estimate has dynamics

$$
d m_{t, 2}=\left[\kappa_{2}\left(\bar{\mu}_{2}-m_{t, 2}\right)+\frac{\beta_{2}\left(m_{1}-m_{2}\right)}{\sigma_{D}^{2}}\right] d t+\frac{\beta_{2}}{\sigma_{D}} d \widehat{W}_{D, t, 1}+\alpha_{s, 2} \sigma_{\mu, 2} d W_{s, t} .
$$

As indicated in Dumas et al. (2009) and Buraschi et al. (2014) the partial differential equation for the joint moment generating function presented above contains the partial differential equation for $H^{m}$, so that the terms related to $H^{m}$ can be cancelled. After plugging in the exponentially affine-quadratic guess we obtain the equation

$$
\begin{aligned}
0= & \left(L_{1}+2 M_{11} m_{1}+2 M_{12} m_{2}\right) \kappa_{1}\left(\bar{\mu}_{1}-m_{1}\right) \\
& +\left(L_{2}+2 M_{12} m_{1}+2 M_{22} m_{2}\right)\left[\kappa_{2}\left(\bar{\mu}_{2}-m_{2}\right)+\frac{\beta_{2}\left(m_{1}-m_{2}\right)}{\sigma_{D}^{2}}\right] \\
& +\frac{1}{2} \chi(\chi-1)\left(\frac{m_{1}-m_{2}}{\sigma_{D}}\right)^{2} \\
& +\frac{1}{2}\left[2 M_{11}+\frac{2\left(1-e^{-\kappa_{1} \tau}\right)}{\kappa_{1}}\left(L_{1}+2 M_{11} m_{1}+2 M_{12} m_{2}\right)+\left(L_{1}+2 M_{11} m_{1}+2 M_{12} m_{2}\right)^{2}\right] \\
& \times\left[\left(\frac{\beta_{1}}{\sigma_{D}}\right)^{2}+\left(\alpha_{s, 1} \sigma_{\mu, 1}\right)^{2}\right] \\
& +\frac{1}{2}\left[2 M_{22}+\left(L_{2}+2 M_{12} m_{1}+2 M_{22} m_{2}\right)^{2}\right]\left[\left(\frac{\beta_{2}}{\sigma_{D}}\right)^{2}+\left(\alpha_{s, 2} \sigma_{\mu, 2}\right)^{2}\right] \\
& +\epsilon\left(L_{1}+2 M_{11} m_{1}+2 M_{12} m_{2}\right) \beta_{1}+\epsilon\left(L_{2}+2 M_{12} m_{1}+2 M_{22} m_{2}\right) \beta_{2}+\epsilon \chi\left(m_{2}-m_{1}\right) \\
& +\left[2 M_{12}+\left(\frac{1-e^{-\kappa_{1} \tau}}{\kappa_{1}}+L_{1}+2 M_{11} m_{1}+2 M_{12} m_{2}\right)\left(L_{2}+2 M_{12} m_{1}+2 M_{22} m_{2}\right)\right] \\
& \times\left[\frac{\beta_{1} \beta_{2}}{\sigma_{D}^{2}}+\left(\alpha_{s, 1} \sigma_{\mu, 1}\right)\left(\alpha_{s, 2} \sigma_{\mu, 2}\right)\right] \\
& +\chi\left(\frac{1-e^{-\kappa_{1} \tau}}{\kappa_{1}}+L_{1}+2 M_{11} m_{1}+2 M_{12} m_{2}\right) \frac{\beta_{1}\left(m_{2}-m_{1}\right)}{\sigma_{D}^{2}} \\
& +\chi\left(L_{2}+2 M_{12} m_{1}+2 M_{22} m_{2}\right) \frac{\beta_{2}\left(m_{2}-m_{1}\right)}{\sigma_{D}^{2}} \\
& -\frac{\partial K}{\partial \tau}-\frac{\partial L_{1}}{\partial \tau} m_{1}-\frac{\partial L_{2}}{\partial \tau} m_{2}-\frac{\partial M_{11}}{\partial \tau} m_{1}^{2}-2 \frac{\partial M_{12}}{\partial \tau} m_{1} m_{2}-\frac{\partial M_{22}}{\partial \tau} m_{2}^{2}
\end{aligned}
$$


Now collect terms in $m_{1}^{2}, m_{2}^{2}, m_{1} m_{2}, m_{1}, M_{2}$, and constants. For the terms in $m_{1}^{2}$ this yields

$$
\begin{aligned}
\frac{\partial M_{11}}{\partial \tau}= & -2 \kappa_{1} M_{11} \\
& +2 M_{12} \frac{\beta_{2}}{\sigma_{D}^{2}} \\
& +\frac{1}{2 \sigma_{D}^{2}} \chi(\chi-1) \\
& +2 M_{11}^{2}\left[\left(\frac{\beta_{1}}{\sigma_{D}}\right)^{2}+\left(\alpha_{s, 1} \sigma_{\mu, 1}\right)^{2}\right] \\
& +2 M_{12}^{2}\left[\left(\frac{\beta_{2}}{\sigma_{D}}\right)^{2}+\left(\alpha_{s, 2} \sigma_{\mu, 2}\right)^{2}\right] \\
& +4 M_{11} M_{12}\left[\frac{\beta_{1} \beta_{2}}{\sigma_{D}^{2}}+\left(\alpha_{s, 1} \sigma_{\mu, 1}\right)\left(\alpha_{s, 2} \sigma_{\mu, 2}\right)\right] \\
& -2 \chi M_{11} \frac{\beta_{1}}{\sigma_{D}^{2}} \\
& -2 \chi M_{12} \frac{\beta_{2}}{\sigma_{D}^{2}} .
\end{aligned}
$$

For the mixed terms $m_{1} m_{2}$ we obtain

$$
\begin{aligned}
\frac{\partial M_{12}}{\partial \tau}= & -M_{12} \kappa_{1} \\
& -M_{12}\left(\kappa_{2}+\frac{\beta_{2}}{\sigma_{D}^{2}}\right)+M_{22} \frac{\beta_{2}}{\sigma_{D}^{2}} \\
& -\frac{1}{2} \chi(\chi-1) \frac{1}{\sigma_{D}^{2}} \\
& +2 M_{11} M_{12}\left[\left(\frac{\beta_{1}}{\sigma_{D}}\right)^{2}+\left(\alpha_{s, 1} \sigma_{\mu, 1}\right)^{2}\right] \\
& +2 M_{12} M_{22}\left[\left(\frac{\beta_{2}}{\sigma_{D}}\right)^{2}+\left(\alpha_{s, 2} \sigma_{\mu, 2}\right)^{2}\right] \\
& +2\left(M_{11} M_{22}+M_{12} M_{12}\right)\left[\frac{\beta_{1} \beta_{2}}{\sigma_{D}^{2}}+\left(\alpha_{s, 1} \sigma_{\mu, 1}\right)\left(\alpha_{s, 2} \sigma_{\mu, 2}\right)\right] \\
& +\chi\left(M_{11}-M_{12}\right) \frac{\beta_{1}}{\sigma_{D}^{2}} \\
& +\chi\left(M_{12}-M_{22}\right) \frac{\beta_{2}}{\sigma_{D}^{2}},
\end{aligned}
$$


and for terms in $m_{2}^{2}$ the ordinary differential equation reads

$$
\begin{aligned}
\frac{\partial M_{22}}{\partial \tau}= & -2 M_{22}\left(\kappa_{2}+\frac{\beta_{2}}{\sigma_{D}^{2}}\right) \\
& +\frac{1}{2} \chi(\chi-1) \frac{1}{\sigma_{D}^{2}} \\
& +2 M_{12}^{2}\left[\left(\frac{\beta_{1}}{\sigma_{D}}\right)^{2}+\left(\alpha_{s, 1} \sigma_{\mu, 1}\right)^{2}\right] \\
& +2 M_{22}^{2}\left[\left(\frac{\beta_{2}}{\sigma_{D}}\right)^{2}+\left(\alpha_{s, 2} \sigma_{\mu, 2}\right)^{2}\right] \\
& +4 M_{12} M_{22}\left[\frac{\beta_{1} \beta_{2}}{\sigma_{D}^{2}}+\left(\alpha_{s, 1} \sigma_{\mu, 1}\right)\left(\alpha_{s, 2} \sigma_{\mu, 2}\right)\right] \\
& +2 \chi M_{12} \frac{\beta_{1}}{\sigma_{D}^{2}} \\
& +2 \chi M_{22} \frac{\beta_{2}}{\sigma_{D}^{2}} .
\end{aligned}
$$

Now define the matrix $M$ as

$$
M=\left(\begin{array}{ll}
M_{11} & M_{12} \\
M_{12} & M_{11}
\end{array}\right)
$$

and the matrices $V, U$, and $N$ as

$$
\begin{gathered}
V=\left(\begin{array}{cc}
-\left(\kappa_{1}+\chi \frac{\beta_{1}}{\sigma_{D}^{2}}\right) & (1-\chi) \frac{\beta_{2}}{\sigma_{D}^{2}} \\
\chi \frac{\beta_{1}}{\sigma_{D}^{2}} & -\left(\kappa_{2}+\frac{\beta_{2}}{\sigma_{D}^{2}}\right)+\chi \frac{\beta_{2}}{\sigma_{D}^{2}}
\end{array}\right) \\
U=\frac{1}{2 \sigma_{D}^{2}}\left(\begin{array}{cc}
\chi(\chi-1) & -\chi(\chi-1) \\
-\chi(\chi-1) & \chi(\chi-1)
\end{array}\right) \\
N=-2\left(\begin{array}{cc}
\left(\frac{\beta_{1}}{\sigma_{D}}\right)^{2}+\left(\alpha_{s, 1} \sigma_{\mu, 1}\right)^{2} & \frac{\beta_{1} \beta_{2}}{\sigma_{D}^{2}}+\left(\alpha_{s, 1} \sigma_{\mu, 1}\right)\left(\alpha_{s, 2} \sigma_{\mu, 2}\right) \\
\frac{\beta_{1} \beta_{2}}{\sigma_{D}^{2}}+\left(\alpha_{s, 1} \sigma_{\mu, 1}\right)\left(\alpha_{s, 2} \sigma_{\mu, 2}\right) & \left(\frac{\beta_{2}}{\sigma_{D}}\right)^{2}+\left(\alpha_{s, 2} \sigma_{\mu, 2}\right)^{2}
\end{array}\right) .
\end{gathered}
$$

The $M$ satisfies the matrix Ricatti equation

$$
\frac{\partial M}{\partial \tau}=V M+M V^{\prime}+U-M N M
$$


Moving on to the linear terms, we get for $m_{1}$

$$
\begin{aligned}
\frac{\partial L_{1}}{\partial \tau}= & -\kappa_{1} L_{1}+2 M_{11} \kappa_{1} \bar{\mu}_{1} \\
& +2 M_{12} \kappa_{2} \bar{\mu}_{2}+L_{2} \frac{\beta_{2}}{\sigma_{D}^{2}} \\
& +\left(\frac{2\left(1-e^{-\kappa_{1} \tau}\right)}{\kappa_{1}} M_{11}+2 M_{11} L_{1}\right)\left[\left(\frac{\beta_{1}}{\sigma_{D}}\right)^{2}+\left(\alpha_{s, 1} \sigma_{\mu, 1}\right)^{2}\right] \\
& +2 M_{12} L_{2}\left[\left(\frac{\beta_{2}}{\sigma_{D}}\right)^{2}+\left(\alpha_{s, 2} \sigma_{\mu, 2}\right)^{2}\right] \\
& +2 \epsilon \beta_{1} M_{11}+2 \epsilon \beta_{2} M_{12}-\epsilon \chi \\
& +\left(2 M_{12} \frac{1-e^{-\kappa_{1} \tau}}{\kappa_{1}}+2 M_{11} L_{2}+2 M_{12} L_{1}\right)\left[\frac{\beta_{1} \beta_{2}}{\sigma_{D}^{2}}+\left(\alpha_{s, 1} \sigma_{\mu, 1}\right)\left(\alpha_{s, 2} \sigma_{\mu, 2}\right)\right] \\
& -\chi\left(\frac{1-e^{-\kappa_{1} \tau}}{\kappa_{1}}+L_{1}\right) \frac{\beta_{1}}{\sigma_{D}^{2}} \\
& -\chi L_{2} \frac{\beta_{2}}{\sigma_{D}^{2}} .
\end{aligned}
$$

Similarly, for $m_{2}$

$$
\begin{aligned}
\frac{\partial L_{2}}{\partial \tau}= & 2 M_{12} \kappa_{1} \bar{\mu}_{1} \\
& +2 M_{22} \kappa_{2} \bar{\mu}_{2}-L_{2}\left(\kappa_{2}+\frac{\beta_{2}}{\sigma_{D}^{2}}\right) \\
& +\left(\frac{2\left(1-e^{-\kappa_{1} \tau}\right)}{\kappa_{1}} M_{12}+2 M_{12} L_{1}\right)\left[\left(\frac{\beta_{1}}{\sigma_{D}}\right)^{2}+\left(\alpha_{s, 1} \sigma_{\mu, 1}\right)^{2}\right] \\
& +2 M_{22} L_{2}\left[\left(\frac{\beta_{2}}{\sigma_{D}}\right)^{2}+\left(\alpha_{s, 2} \sigma_{\mu, 2}\right)^{2}\right] \\
& +2 \epsilon \beta_{1} M_{12}+2 \epsilon \beta_{2} M_{22}+\epsilon \chi \\
& +\left(2 M_{22} \frac{1-e^{-\kappa_{1} \tau}}{\kappa_{1}}+2 M_{12} L_{2}+2 M_{22} L_{1}\right)\left[\frac{\beta_{1} \beta_{2}}{\sigma_{D}^{2}}+\left(\alpha_{s, 1} \sigma_{\mu, 1}\right)\left(\alpha_{s, 2} \sigma_{\mu, 2}\right)\right] \\
& +\chi\left(\frac{1-e^{-\kappa_{1} \tau}}{\kappa_{1}}+L_{1}\right) \frac{\beta_{1}}{\sigma_{D}^{2}} \\
& +\chi L_{2} \frac{\beta_{2}}{\sigma_{D}^{2}} .
\end{aligned}
$$

Define the vector $L \equiv\left(L_{1}, L_{2}\right)^{\prime}$. Then $L$ satisfies the ordinary differential 
equation

$$
\frac{\partial L}{\partial \tau}=2 M b+(V-M N) L+a
$$

with boundary condition $L(0)=0$ and

$$
\begin{aligned}
a \equiv\left(\begin{array}{c}
-\chi\left(\epsilon+\frac{1-e^{-\kappa_{1} \tau}}{\kappa_{1}} \frac{\beta_{1}}{\sigma_{D}^{2}}\right) \\
\chi\left(\epsilon+\frac{1-e^{-\kappa_{1} \tau}}{\kappa_{1}} \frac{\beta_{1}}{\sigma_{D}^{2}}\right)
\end{array}\right) \\
b \equiv\left(\begin{array}{c}
\kappa_{1} \bar{\mu}_{1}+\epsilon \beta_{1}+\frac{1-e^{-\kappa_{1} \tau}}{\kappa_{1}}\left[\left(\frac{\beta_{1}}{\sigma_{D}}\right)^{2}+\left(\alpha_{s, 1} \sigma_{\mu, 1}\right)^{2}\right] \\
\kappa_{2} \bar{\mu}_{2}+\epsilon \beta_{2}+\frac{1-e^{-\kappa_{1} \tau}}{\kappa_{1}}\left[\frac{\beta_{1} \beta_{2}}{\sigma_{D}^{2}}+\left(\alpha_{s, 1} \sigma_{\mu, 1}\right)\left(\alpha_{s, 2} \sigma_{\mu, 2}\right)\right]
\end{array}\right) .
\end{aligned}
$$

Finally, for the constant terms we obtain the differential equation

$$
\frac{\partial K}{\partial \tau}=L^{\prime} b-\frac{1}{4} L^{\prime} N L-\frac{1}{2} \operatorname{trace}(M N)
$$

We solve the differential equations Appendix A.2 and Appendix A.3 numerically using standard solvers like ode45 in MATLAB and then numerical integrate Appendix A.4.

Given the functions $H^{m}$ and $H^{g}$ the equilibrium stock price $S_{t}$ can be computed as

$$
\begin{aligned}
S_{t}= & D_{t} E^{\mathbb{P}^{1}}\left[\int_{t}^{\infty} e^{-\delta(u-t)} D_{u}\left(\frac{C_{u, 1}}{C_{t, 1}}\right)^{-\nu} d u\right] \\
= & D_{t} \int_{t}^{\infty} e^{-\delta(u-t)} \omega\left(\eta_{t}\right)^{\nu} H^{m}\left(1-\nu ; t, u, m_{1, t}\right) \\
& \sum_{j=0}^{\nu}\left(\begin{array}{c}
\nu \\
j
\end{array}\right)\left(\frac{1-\omega\left(\eta_{t}\right)}{\omega\left(\eta_{t}\right)}\right)^{j} H^{g}\left(1-\nu, \frac{j}{\nu} ; t, u, m_{t, 1}, m_{t, 2}\right) d u .
\end{aligned}
$$

Appendix A.2. Growth Condition for the Stock Price

Under the assumption that investor $i$ survives and thus consumes the total dividend in the long-run, the growth condition holds (and the stock price will not explode), when the subjective expectation of discounted future dividends remains finite:

$$
E_{t}^{\mathbb{P}^{i}}\left[\int_{t}^{\infty} D_{u} \frac{\xi_{u}^{i}}{\xi_{t}^{i}} d u\right]<\infty
$$


with $\xi_{i}$ as investor $i$ 's pricing kernel. Under CRRA preferences the pricing kernel is given by the ratio of marginal utilities, i.e., $\xi_{t}^{i}=e^{-\delta t}\left(\frac{D_{t}}{D_{0}}\right)^{-\nu}$, so that the condition becomes

$$
E_{t}^{\mathbb{P}^{i}}\left[\int_{t}^{\infty} e^{-\delta(u-t)} D_{u}^{1-\nu} D_{t}^{\nu} d u\right]<\infty .
$$

The expectation follows from Equation Appendix A.1 with $\epsilon \equiv 1-\nu$. The critical terms for the growth condition to hold are the terms in the exponential which are in $u-t$. Restricting these terms yields the growth condition for investor $i=1,2$ :

$$
(1-\nu)\left(\bar{\mu}_{i}-\frac{1}{2} \sigma_{D}^{2}\right)+\frac{(1-\nu)^{2}}{2}\left[\left(\sigma_{D}+\frac{\beta_{i}}{\kappa_{i} \sigma_{D}}\right)^{2}+\left(\frac{\alpha_{s, i} \sigma_{\mu, i}}{\kappa_{i}}\right)^{2}\right]<\delta
$$

(Appendix A.5)

For the model to be well-defined, condition Appendix A.5 has to hold for investors 1 and 2. Otherwise, we exclude the respective parameter combination from further consideration.

Appendix A.3. Consumption Sharing Rule

Proposition 1 (Consumption Sharing Rule) Investor 2's consumption share $\omega_{t} \equiv \frac{C_{t, 2}}{C_{t}}$ is given by

$$
\omega_{t}=\frac{\left(\frac{\eta_{t}}{\phi_{2}}\right)^{\frac{1}{\nu}}}{\left(\frac{\eta_{t}}{\phi_{2}}\right)^{\frac{1}{\nu}}+\left(\frac{1}{\phi_{1}}\right)^{\frac{1}{\nu}}}
$$

where $\phi_{1}$ and $\phi_{2}$ are the Lagrange multipliers for the budget restrictions of investor 1 and 2 , respectively, and where $\eta$ is the Radon-Nikodym-derivative $\eta=\frac{d \mathbb{P}_{2}}{d \mathbb{P}_{1}}$.

To see this, note first that the optimality conditions for the investors' consumption are given as

$$
\begin{aligned}
e^{-\delta t} C_{t, 1}^{-\nu} & =\phi_{1} \xi_{t, 1} \\
e^{-\rho t} C_{t, 2}^{-\nu} & =\phi_{2} \xi_{t, 2}
\end{aligned}
$$


where $\phi_{1}$ and $\phi_{2}$ are the Lagrange multipliers for the budget restriction and where $\xi_{i}$ is investor $i$ 's stochastic discount factor. Taking the ratio of the two expressions gives

$$
C_{t, 1}^{-\nu}=C_{t, 2}^{-\nu} \frac{\phi_{1}}{\phi_{2}} \eta_{t},
$$

which can be solved for $C_{t, 2}$, yielding

$$
C_{t, 2}=C_{t, 1}\left(\frac{\phi_{1}}{\phi_{2}} \eta_{t}\right)^{\frac{1}{\nu}} .
$$

Together the two investors have to consume aggregate endowment, so that

$$
C_{t, 1}\left[1+\left(\frac{\phi_{1}}{\phi_{2}} \eta_{t}\right)^{\frac{1}{\nu}}\right]=C_{t}
$$

which, together with $C_{t, 1} / C_{t} \equiv 1-\omega_{t}$, yields the proposition.

\section{Appendix A.4. Dynamics of Consumption}

Proposition 2 (Consumption dynamics) The dynamics of the investors' individual consumption levels are given by

$$
\begin{aligned}
\frac{d C_{t, 1}}{C_{t, 1}} & =\left[m_{t, 1}+\frac{\omega_{t}}{\nu}\left(\frac{1+\nu-2\left(1-\omega_{t}\right)}{2 \nu} \frac{g_{t}^{2}}{\sigma_{D}^{2}}+g_{t}\right)\right] d t+\left(\sigma_{D}+\frac{\omega_{t}}{\nu} \frac{g_{t}}{\sigma_{D}}\right) d \widehat{W}_{D, t, 1} \\
\frac{d C_{2}}{C_{2}} & =\left[m_{t, 2}+\frac{1-\omega_{t}}{\nu}\left(\frac{1+\nu-2 \omega_{t}}{2 \nu} \frac{g_{t}^{2}}{\sigma_{D}^{2}}-g_{t}\right)\right] d t+\left(\sigma_{D}-\frac{1-\omega_{t}}{\nu} \frac{g_{t}}{\sigma_{D}}\right) d \widehat{W}_{D, t, 2} .
\end{aligned}
$$

The partial derivatives of $\omega$ with respect to $\eta$ are

$$
\frac{\partial \omega}{\partial \eta}=(1-\omega) \omega \frac{1}{\nu \eta}
$$

and

$$
\frac{\partial^{2} \omega}{\partial \eta^{2}}=(1-\omega) \omega_{2} \frac{1-\nu-2 \omega}{\nu^{2} \eta^{2}} .
$$

Together with the dynamics of $\omega$ given by

$$
\begin{aligned}
d \omega_{t} & =\frac{\left(1-\omega_{t}\right) \omega_{t}}{\nu}\left[\frac{1-\nu-2 \omega_{t}}{2 \nu} \frac{g_{t}^{2}}{\sigma_{D}^{2}} d t-\frac{g_{t}}{\sigma_{D}} d \widehat{W}_{D, t, 1}\right] \\
& =\frac{\left(1-\omega_{t}\right) \omega_{t}}{\nu}\left[\left(\frac{1-2 \omega_{t}}{2 \nu} \frac{g_{t}^{2}}{\sigma_{D}^{2}}+\frac{g_{t}\left(g_{t, 1}+g_{t, 2}\right)}{2 \sigma_{D}^{2}}\right) d t-\frac{g_{t}}{\sigma_{D}} d W_{D, t}\right]
\end{aligned}
$$


one obtains dynamics of investor 2's consumption

$$
\begin{aligned}
\frac{d C_{t, 2}}{C_{t, 2}}= & \frac{d \omega_{t}}{\omega_{t}}+\frac{d C_{t}}{C_{t}}+\frac{d \omega_{t}}{\omega_{t}} \frac{d C_{t}}{C_{t}} \\
= & {\left[m_{t, 2}+\frac{1-\omega_{t}}{\nu}\left(\frac{1+\nu-2 \omega_{t}}{2 \nu} \frac{g_{t}^{2}}{\sigma_{D}^{2}}-g_{t}\right)\right] d t+\left(\sigma_{D}-\frac{1-\omega_{t}}{\nu} \frac{g_{t}}{\sigma_{D}}\right) d \widehat{W}_{D, t, 2} } \\
= & {\left[\mu_{t}+\frac{1-\omega_{t}}{\nu}\left(\frac{1-2 \omega_{t}}{2 \nu} \frac{g_{t}^{2}}{\sigma_{D}^{2}}+\frac{1}{2} \frac{\left(g_{t, 1}-g_{t, 2}\right)\left(g_{t, 1}+g_{t, 2}\right)}{\sigma_{D}^{2}}-g_{t}\right)\right] d t } \\
& +\left(\sigma_{D}-\frac{1-\omega_{t}}{\gamma} \frac{g_{t}}{\sigma_{D}}\right) d W_{D, t} .
\end{aligned}
$$

Appendix A.5. Risk-free rate and market prices of risk

Proposition 3 (Risk-free rate and market prices of risk) The risk-free rate is

$$
r_{t}=\delta+\nu\left(\left(1-\omega_{t}\right) m_{t, 1}+\omega_{t} m_{t, 2}\right)+\left(1-\omega_{t}\right) \omega_{t} \frac{\nu-1}{2 \nu} \frac{g_{t}^{2}}{\sigma_{D}^{2}}-0.5 \nu(\nu+1) \sigma_{D}^{2} .
$$

The market prices of dividend risk as perceived by investor 1 and 2 are

$$
\begin{aligned}
& \lambda_{t, 1}=\nu \sigma_{D}+\omega_{t} \frac{g_{t}}{\sigma_{D}} \\
& \lambda_{t, 2}=\nu \sigma_{D}-\left(1-\omega_{t}\right) \frac{g_{t}}{\sigma_{D}} .
\end{aligned}
$$

The market prices of dividend risk under the true measure is

$$
\bar{\lambda}_{t}=\nu \sigma_{D}-\frac{\left(1-\omega_{t}\right) g_{t, 1}+\omega_{t, 2} g_{t, 2}}{\sigma_{D}}
$$

Investor 1's stochastic discount factor is given as

$$
\xi_{t, 1}=e^{-\delta t}\left(\frac{C_{t, 1}}{C_{0,1}}\right)^{-\nu}
$$

with dynamics

$$
\frac{d \xi_{t, 1}}{\xi_{t, 1}}=-\delta d t-\nu \frac{d C_{t, 1}}{C_{t, 1}}+\frac{1}{2} \nu(\nu+1)\left(\frac{d C_{t, 1}}{C_{t, 1}}\right)^{2}
$$


Plugging in the consumption dynamics derived in Appendix Appendix A.4 one obtains

$$
\begin{aligned}
\frac{d \xi_{t, 1}}{\xi_{t, 1}}= & -\delta d t-\nu\left[m_{t, 1}+\frac{\omega_{t}}{\nu}\left(\frac{1+\nu-2\left(1-\omega_{t}\right)}{2 \nu} \frac{g_{t}^{2}}{\sigma_{D}^{2}}+g_{t}\right)\right] d t-\nu\left(\sigma_{D}+\frac{\omega_{t}}{\nu} \frac{g_{t}}{\sigma_{D}}\right) d \widehat{W}_{D, t, 1} \\
& +0.5 \nu(\nu+1)\left(\sigma_{D}+\frac{\omega_{t}}{\nu} \frac{g_{t}}{\sigma_{D}}\right)^{2} d t \\
= & -\left[\delta+\nu\left(\left(1-\omega_{t}\right) m_{t, 1}+\omega_{t} m_{t, 2}\right)+\left(1-\omega_{t}\right) \omega_{t} \frac{\nu-1}{2 \nu} \frac{g_{t}^{2}}{\sigma_{D}^{2}}-0.5 \nu(\nu+1) \sigma_{D}^{2}\right] d t \\
& -\left(\nu \sigma_{D}+\omega_{t} \frac{g_{t}}{\sigma_{D}}\right) d \widehat{W}_{D, t, 1} .
\end{aligned}
$$

From this we obtain the risk-free rate $r$ (on which the investors agree) and the subjective market price of risk $\lambda_{1}$ as perceived by investor 1 :

$$
\begin{aligned}
r_{t} & =\delta+\nu\left(\left(1-\omega_{t}\right) m_{t, 1}+\omega_{t} m_{t, 2}\right)+\left(1-\omega_{t}\right) \omega_{t} \frac{\nu-1}{2 \nu} \frac{g_{t}^{2}}{\sigma_{D}^{2}}-0.5 \nu(\nu+1) \sigma_{D}^{2} \\
\lambda_{t, 1} & =\nu \sigma_{D}+\omega_{t} \frac{g_{t}}{\sigma_{D}} .
\end{aligned}
$$

Going through the analogous computations for investor 2 one finds

$$
\lambda_{t, 2}=\nu \sigma_{D}-\left(1-\omega_{t}\right) \frac{g_{t}}{\sigma_{D}} .
$$

Under the true measure the stochastic discount factor is

$$
\xi_{t}=\xi_{t, 1} \frac{d \mathbb{P}_{t, 1}}{d \mathbb{P}_{t}},
$$

where the change of measure is given by

$$
d \frac{d \mathbb{P}_{t, 1}}{d \mathbb{P}_{t}}=\frac{d \mathbb{P}_{t, 1}}{d \mathbb{P}_{t}} \frac{g_{t, 1}}{\sigma_{D}} d W_{D, t}
$$

so that

$$
\begin{aligned}
\frac{d \xi_{t}}{\xi_{t}} & =\frac{d \xi_{t, 1}}{\xi_{t, 1}}+\frac{d \frac{d \mathbb{P}_{t, 1}}{d \mathbb{P}_{t}}}{\frac{d \mathbb{d t}_{t, 1}}{d \mathbb{P}_{t}}}+\frac{d \xi_{t, 1}}{\xi_{t, 1}} \cdot \frac{d \frac{d \mathbb{P}_{t, 1}}{d \mathbb{P}_{t}}}{\frac{d \mathbb{P}_{t, 1}}{d \mathbb{P}_{t}}} \\
& =-r_{t} d t-\left(\nu \sigma_{D}-\frac{\left(1-\omega_{t}\right) g_{t, 1}+\omega_{t} g_{t, 2}}{\sigma_{D}}\right) d W_{D, t}
\end{aligned}
$$


Appendix A.6. Characteristic Function of Consumption Share

The true model is given in Equations (1) to (2). The dynamics of the investors' estimates of the expected growth rate and of the change of measure under the true measure $\mathbb{P}$ are shown in Equations (14) to (15). Together with the guess for the function $H^{\mathbb{P}}\left(m_{1}, m_{2}, \mu, t, u ; \chi\right)$ from 21 this implies the following partial differential equation

$$
\begin{aligned}
0= & \left(\frac{\partial A}{\partial \tau}+\frac{\partial B_{1}}{\partial \tau} m_{1}+\frac{\partial B_{2}}{\partial \tau} m_{2}+\frac{\partial B_{3}}{\partial \tau} \mu\right. \\
+ & \left.\frac{\partial C_{11}}{\partial \tau} m_{1}^{2}+2 \frac{\partial C_{12}}{\partial \tau} m_{1} m_{2}+2 \frac{\partial C_{13}}{\partial \tau} m_{1} \mu+\frac{\partial C_{22}}{\partial \tau} m_{2}^{2}+2 \frac{\partial C_{23}}{\partial \tau} m_{2} \mu+\frac{\partial C_{33}}{\partial \tau} \mu^{2}\right) \\
+ & {\left[B_{1}+2\left(C_{11} m_{1}+C_{12} m_{2}+C_{13} \mu\right)\right]\left[\kappa_{1}\left(\bar{\mu}_{1}-m_{1}\right)+\left(\frac{\beta_{1}}{\sigma_{D}^{2}}\right)\left(\mu-m_{1}\right)\right] } \\
+ & {\left[B_{2}+2\left(C_{12} m_{1}+C_{22} m_{2}+C_{23} \mu\right)\right]\left[\kappa_{2}\left(\bar{\mu}_{2}-m_{2}\right)+\left(\frac{\beta_{2}}{\sigma_{D}^{2}}\right)\left(\mu-m_{2}\right)\right] } \\
+ & {\left[B_{3}+2\left(C_{13} m_{1}+C_{23} m_{2}+C_{33} \mu\right)\right] \kappa(\bar{\mu}-\mu) } \\
+ & \chi \frac{\left(m_{1}-\mu\right)\left(m_{1}-m_{2}\right)}{\sigma_{D}^{2}} \\
+ & \frac{1}{2}\left\{2 C_{11}+\left[B_{1}+2\left(C_{11} m_{1}+C_{12} m_{2}+C_{13} \mu\right)\right]^{2}\right\}\left[\left(\frac{\beta_{1}}{\sigma_{D}}\right)^{2}+\left(\alpha_{s, 1} \sigma_{\mu, 1}\right)^{2}\right] \\
+ & \frac{1}{2}\left\{2 C_{22}+\left[B_{2}+2\left(C_{12} m_{1}+C_{22} m_{2}+C_{23} \mu\right)\right]^{2}\right\}\left[\left(\frac{\beta_{2}}{\sigma_{D}}\right)^{2}+\left(\alpha_{s, 2} \sigma_{\mu, 2}\right)^{2}\right] \\
+ & \frac{1}{2}\left\{2 C_{33}+\left[B_{3}+2\left(C_{13} m_{1}+C_{23} m_{2}+C_{33} \mu\right)\right]^{2}\right\} \sigma_{\mu}^{2} \\
+ & \frac{1}{2} \chi(\chi-1)\left(\frac{m_{1}-m_{2}}{\sigma_{D}}\right)^{2} \\
+ & \chi\left[B_{1}+2\left(C_{11} m_{1}+C_{12} m_{2}+C_{13} \mu\right)\right] \frac{\beta_{1}\left(m_{2}-m_{1}\right)}{\sigma_{D}^{2}} \\
+ & \chi\left[B_{2}+2\left(C_{12} m_{1}+C_{22} m_{2}+C_{23} \mu\right)\right] \frac{\beta_{2}\left(m_{2}-m_{1}\right)}{\sigma_{D}^{2}} \\
+ & \chi\left[B_{3}+2\left(C_{13} m_{1}+C_{23} m_{2}+C_{33} \mu\right)\right] \alpha_{D} \sigma_{\mu} \frac{m_{2}-m_{1}}{\sigma_{D}} \\
+ & \left\{2 C_{12}+\left[B_{1}+2\left(C_{11} m_{1}+C_{12} m_{2}+C_{13} \mu\right)\right]\left[B_{2}+2\left(C_{12} m_{1}+C_{22} m_{2}+C_{23} \mu\right)\right]\right\} \\
& \times\left[\frac{\beta_{1} \beta_{2}}{\sigma_{D}^{2}}+\alpha_{s, 1} \alpha_{s, 2} \sigma_{\mu, 1} \sigma_{\mu, 2}\right] \\
+ & \left\{2 C_{13}+\left[B_{1}+2\left(C_{11} m_{1}+C_{12} m_{2}+C_{13} \mu\right)\right]\left[B_{3}+2\left(C_{13} m_{1}+C_{23} m_{2}+C_{33} \mu\right)\right]\right\} \\
& \times\left[\frac{\beta_{1}}{\sigma_{D}} \alpha_{D} \sigma_{\mu}+\alpha_{s} \alpha_{s, 1} \sigma_{\mu} \sigma_{\mu, 1}\right] \\
+ & {\left[2 C_{23}+\left[B_{2}+2\left(C_{12} m_{1}+C_{22} m_{2}+C_{D} \sigma_{\mu}+\alpha_{s} \alpha_{s, 2} \sigma_{\mu} \sigma_{\mu, 2}\right],\right.\right.} \\
\sigma_{D} & \\
&
\end{aligned}
$$

where $\beta_{i} \equiv \gamma_{i}+\alpha_{D, i} \sigma_{D} \sigma_{\mu, i}$. 
To obtain a solution, collect squared and linear terms in the state variables $m_{1}$, $m_{2}$, and $\mu$ as well as the constants. Collecting terms in $m_{1}^{2}$ yields the ordinary differential equation

$$
\begin{aligned}
\frac{\partial C_{11}}{\partial \tau}= & -2\left(\kappa_{1}+\frac{\beta_{1}}{\sigma_{D}^{2}}\right) C_{11}+\frac{\chi}{\sigma_{D}^{2}}+2 C_{11}^{2}\left[\left(\frac{\beta_{1}}{\sigma_{D}}\right)^{2}+\left(\alpha_{s, 1} \sigma_{\mu, 1}\right)^{2}\right] \\
& +2 C_{12}^{2}\left[\left(\frac{\beta_{2}}{\sigma_{D}}\right)^{2}+\left(\alpha_{s, 2} \sigma_{\mu, 2}\right)^{2}\right]+2 C_{13}^{2} \sigma_{\mu}^{2}+\frac{\chi(\chi-1)}{2 \sigma_{D}^{2}} \\
& -2 \chi C_{11} \frac{\beta_{1}}{\sigma_{D}^{2}}-2 \chi C_{12} \frac{\beta_{2}}{\sigma_{D}^{2}}-2 \chi C_{13} \frac{\alpha_{D} \sigma_{\mu}}{\sigma_{D}} \\
& +4 C_{11} C_{12}\left[\frac{\beta_{1} \beta_{2}}{\sigma_{D}^{2}}+\alpha_{s, 1} \alpha_{s, 2} \sigma_{\mu, 1} \sigma_{\mu, 2}\right]+4 C_{11} C_{13}\left[\frac{\beta_{1} \alpha_{D} \sigma_{\mu}}{\sigma_{D}}+\alpha_{s} \alpha_{s, 1} \sigma_{\mu} \sigma_{\mu, 1}\right] \\
& +4 C_{12} C_{13}\left[\frac{\beta_{2} \alpha_{D} \sigma_{\mu}}{\sigma_{D}}+\alpha_{s} \alpha_{s, 2} \sigma_{\mu} \sigma_{\mu, 2}\right]
\end{aligned}
$$

Analogously we obtain for terms in $m_{2}^{2}$

$$
\begin{aligned}
\frac{\partial C_{22}}{\partial \tau}= & -2\left(\kappa_{2}+\frac{\beta_{2}}{\sigma_{D}^{2}}\right) C_{22}+2 C_{12}^{2}\left[\left(\frac{\beta_{1}}{\sigma_{D}}\right)^{2}+\left(\alpha_{s, 1} \sigma_{\mu, 1}\right)^{2}\right] \\
& +2 C_{22}^{2}\left[\left(\frac{\beta_{2}}{\sigma_{D}}\right)^{2}+\left(\alpha_{s, 2} \sigma_{\mu, 2}\right)^{2}\right]+2 C_{23}^{2} \sigma_{\mu}^{2}+\frac{\chi(\chi-1)}{2 \sigma_{D}^{2}} \\
& +2 \chi C_{12} \frac{\beta_{1}}{\sigma_{D}^{2}}+2 \chi C_{22} \frac{\beta_{2}}{\sigma_{D}^{2}}+2 \chi C_{23} \frac{\alpha_{D} \sigma_{\mu}}{\sigma_{D}^{2}} \\
& +4 C_{12} C_{22}\left[\frac{\beta_{1} \beta_{2}}{\sigma_{D}^{2}}+\alpha_{s, 1} \alpha_{s, 2} \sigma_{\mu, 1} \sigma_{\mu, 2}\right]+4 C_{12} C_{23}\left[\frac{\beta_{1} \alpha_{D} \sigma_{\mu}}{\sigma_{D}}+\alpha_{s} \alpha_{s, 1} \sigma_{\mu} \sigma_{\mu, 1}\right] \\
& +4 C_{22} C_{23}\left[\frac{\beta_{2} \alpha_{D} \sigma_{\mu}}{\sigma_{D}}+\alpha_{s} \alpha_{s, 2} \sigma_{\mu} \sigma_{\mu, 2}\right] .
\end{aligned}
$$

For terms in $\mu^{2}$ the equation becomes

$$
\begin{aligned}
\frac{\partial C_{33}}{\partial \tau}= & 2 C_{13} \frac{\beta_{1}}{\sigma_{D}^{2}}+2 C_{23} \frac{\beta_{2}}{\sigma_{D}^{2}}-2 C_{33} \kappa \\
& +2 C_{13}^{2}\left[\left(\frac{\beta_{1}}{\sigma_{D}}\right)^{2}+\left(\alpha_{s, 1} \sigma_{\mu, 1}\right)^{2}\right]+2 C_{23}^{2}\left[\left(\frac{\beta_{2}}{\sigma_{D}}\right)^{2}+\left(\alpha_{s, 2} \sigma_{\mu, 2}\right)^{2}\right]+2 C_{33}^{2} \sigma_{\mu}^{2} \\
& +4 C_{13} C_{23}\left[\frac{\beta_{1} \beta_{2}}{\sigma_{D}^{2}}+\alpha_{s, 1} \alpha_{s, 2} \sigma_{\mu, 1} \sigma_{\mu, 2}\right] \\
& +4 C_{13} C_{33}\left[\frac{\beta_{1} \alpha_{D} \sigma_{\mu}}{\sigma_{D}}+\alpha_{s} \alpha_{s, 1} \sigma_{\mu} \sigma_{\mu, 1}\right]+4 C_{23} C_{33}\left[\frac{\beta_{2} \alpha_{D} \sigma_{\mu}}{\sigma_{D}}+\alpha_{s} \alpha_{s, 2} \sigma_{\mu} \sigma_{\mu, 2}\right]
\end{aligned}
$$


For the mixed terms in $m_{1} m_{2}$ we obtain

$$
\begin{aligned}
2 \frac{\partial C_{12}}{\partial \tau}= & -2\left(\kappa_{1}+\frac{\beta_{1}}{\sigma_{D}^{2}}\right) C_{12}-2\left(\kappa_{2}+\frac{\beta_{2}}{\sigma_{D}^{2}}\right) C_{12}-\frac{\chi}{\sigma_{D}^{2}} \\
& +4 C_{11} C_{12}\left[\left(\frac{\beta_{1}}{\sigma_{D}}\right)^{2}+\left(\alpha_{s, 1} \sigma_{\mu, 1}\right)^{2}\right]+4 C_{12} C_{22}\left[\left(\frac{\beta_{2}}{\sigma_{D}}\right)^{2}+\left(\alpha_{s, 2} \sigma_{\mu, 2}\right)^{2}\right]+4 C_{13} C_{23} \sigma_{\mu}^{2} \\
& -\frac{\chi(\chi-1)}{\sigma_{D}^{2}} \\
& +2 \chi C_{11} \frac{\beta_{1}}{\sigma_{D}^{2}}-2 \chi C_{12} \frac{\beta_{1}}{\sigma_{D}^{2}}+2 \chi C_{12} \frac{\beta_{2}}{\sigma_{D}^{2}}-2 \chi C_{22} \frac{\beta_{2}}{\sigma_{D}^{2}}+2 \chi C_{13} \frac{\alpha_{D} \sigma_{\mu}}{\sigma_{D}}-2 \chi C_{23} \frac{\alpha_{D} \sigma_{\mu}}{\sigma_{D}} \\
& +4 C_{11} C_{22}\left[\frac{\beta_{1} \beta_{2}}{\sigma_{D}^{2}}+\alpha_{s, 1} \alpha_{s, 2} \sigma_{\mu, 1} \sigma_{\mu, 2}\right]+4 C_{12}^{2}\left[\frac{\beta_{1} \beta_{2}}{\sigma_{D}^{2}}+\alpha_{s, 1} \alpha_{s, 2} \sigma_{\mu, 1} \sigma_{\mu, 2}\right] \\
& +4 C_{11} C_{23}\left[\frac{\beta_{1} \alpha_{D} \sigma_{\mu}}{\sigma_{D}}+\alpha_{s} \alpha_{s, 1} \sigma_{\mu} \sigma_{\mu, 1}\right]+4 C_{12} C_{13}\left[\frac{\beta_{1} \alpha_{D} \sigma_{\mu}}{\sigma_{D}}+\alpha_{s} \alpha_{s, 1} \sigma_{\mu} \sigma_{\mu, 1}\right] \\
& +4 C_{12} C_{23}\left[\frac{\beta_{2} \alpha_{D} \sigma_{\mu}}{\sigma_{D}}+\alpha_{s} \alpha_{s, 2} \sigma_{\mu} \sigma_{\mu, 2}\right]+4 C_{22} C_{13}\left[\frac{\beta_{2} \alpha_{D} \sigma_{\mu}}{\sigma_{D}}+\alpha_{s} \alpha_{s, 2} \sigma_{\mu} \sigma_{\mu, 2}\right],
\end{aligned}
$$

while for terms in $m_{1} \mu$ we get

$$
\begin{aligned}
2 \frac{\partial C_{13}}{\partial \tau}= & 2 \frac{\beta_{1}}{\sigma_{D}^{2}} C_{11}-2\left(\kappa_{1}+\frac{\beta_{1}}{\sigma_{D}^{2}}\right) C_{13}-2 C_{13} \kappa-\frac{\chi}{\sigma_{D}^{2}} \\
& +4 C_{11} C_{13}\left[\left(\frac{\beta_{1}}{\sigma_{D}}\right)^{2}+\left(\alpha_{s, 1} \sigma_{\mu, 1}\right)^{2}\right]+4 C_{12} C_{23}\left[\left(\frac{\beta_{2}}{\sigma_{D}}\right)^{2}+\left(\alpha_{s, 2} \sigma_{\mu, 2}\right)^{2}\right]+4 C_{13} C_{33} \sigma_{\mu}^{2} \\
& -2 \chi C_{13} \frac{\beta_{1}}{\sigma_{D}^{2}}-2 \chi C_{23} \frac{\beta_{2}}{\sigma_{D}^{2}}-2 \chi C_{33} \frac{\alpha_{D} \sigma_{\mu}}{\sigma_{D}} \\
& +4 C_{11} C_{23}\left[\frac{\beta_{1} \beta_{2}}{\sigma_{D}^{2}}+\alpha_{s, 1} \alpha_{s, 2} \sigma_{\mu, 1} \sigma_{\mu, 2}\right]+4 C_{13} C_{12}\left[\frac{\beta_{1} \beta_{2}}{\sigma_{D}^{2}}+\alpha_{s, 1} \alpha_{s, 2} \sigma_{\mu, 1} \sigma_{\mu, 2}\right] \\
& +4 C_{11} C_{33}\left[\frac{\beta_{1} \alpha_{D} \sigma_{\mu}}{\sigma_{D}}+\alpha_{s} \alpha_{s, 1} \sigma_{\mu} \sigma_{\mu, 1}\right]+4 C_{13}^{2}\left[\frac{\beta_{1} \alpha_{D} \sigma_{\mu}}{\sigma_{D}}+\alpha_{s} \alpha_{s, 1} \sigma_{\mu} \sigma_{\mu, 1}\right] \\
& +4 C_{12} C_{33}\left[\frac{\beta_{2} \alpha_{D} \sigma_{\mu}}{\sigma_{D}}+\alpha_{s} \alpha_{s, 2} \sigma_{\mu} \sigma_{\mu, 2}\right]+4 C_{23} C_{13}\left[\frac{\beta_{2} \alpha_{D} \sigma_{\mu}}{\sigma_{D}}+\alpha_{s} \alpha_{s, 2} \sigma_{\mu} \sigma_{\mu, 2}\right]
\end{aligned}
$$


For the terms containing the product $m_{2} \mu$ the differential equation reads as follows:

$$
\begin{aligned}
2 \frac{\partial C_{23}}{\partial \tau}= & 2 \frac{\beta_{1}}{\sigma_{D}^{2}} C_{12}+2 \frac{\beta_{2}}{\sigma_{D}^{2}} C_{22}-2\left(\kappa_{2}+\frac{\beta_{2}}{\sigma_{D}^{2}}\right) C_{23}-2 \kappa C_{23}+\frac{\chi}{\sigma_{D}^{2}} \\
& +4 C_{12} C_{13}\left[\left(\frac{\beta_{1}}{\sigma_{D}}\right)^{2}+\left(\alpha_{s, 1} \sigma_{\mu, 1}\right)^{2}\right]+4 C_{22} C_{23}\left[\left(\frac{\beta_{2}}{\sigma_{D}}\right)^{2}+\left(\alpha_{s, 2} \sigma_{\mu, 2}\right)^{2}\right]+4 C_{23} C_{33} \sigma_{\mu}^{2} \\
& +2 \chi C_{13} \frac{\beta_{1}}{\sigma_{D}^{2}}+2 \chi C_{23} \frac{\beta_{2}}{\sigma_{D}^{2}}+2 \chi C_{33} \frac{\alpha_{D} \sigma_{\mu}}{\sigma_{D}} \\
& +4 C_{12} C_{23}\left[\frac{\beta_{1} \beta_{2}}{\sigma_{D}^{2}}+\alpha_{s, 1} \alpha_{s, 2} \sigma_{\mu, 1} \sigma_{\mu, 2}\right]+4 C_{13} C_{22}\left[\frac{\beta_{1} \beta_{2}}{\sigma_{D}^{2}}+\alpha_{s, 1} \alpha_{s, 2} \sigma_{\mu, 1} \sigma_{\mu, 2}\right] \\
& +4 C_{12} C_{33}\left[\frac{\beta_{1} \alpha_{D} \sigma_{\mu}}{\sigma_{D}}+\alpha_{s} \alpha_{s, 1} \sigma_{\mu} \sigma_{\mu, 1}\right]+4 C_{13} C_{23}\left[\frac{\beta_{1} \alpha_{D} \sigma_{\mu}}{\sigma_{D}}+\alpha_{s} \alpha_{s, 1} \sigma_{\mu} \sigma_{\mu, 1}\right] \\
& +4 C_{22} C_{33}\left[\frac{\beta_{2} \alpha_{D} \sigma_{\mu}}{\sigma_{D}}+\alpha_{s} \alpha_{s, 2} \sigma_{\mu} \sigma_{\mu, 2}\right]+4 C_{23}^{2}\left[\frac{\beta_{2} \alpha_{D} \sigma_{\mu}}{\sigma_{D}}+\alpha_{s} \alpha_{s, 2} \sigma_{\mu} \sigma_{\mu, 2}\right]
\end{aligned}
$$

From terms linear in $m_{1}, m_{2}$, and $\mu$ one obtains the following three equations

$$
\begin{aligned}
\frac{\partial B_{1}}{\partial \tau}= & -\left(\kappa_{1}+\frac{\beta_{1}}{\sigma_{D}^{2}}\right) B_{1}+2 C_{11} \kappa_{1} \bar{\mu}_{1}+2 C_{12} \kappa_{2} \bar{\mu}_{2}+2 C_{13} \kappa \bar{\mu} \\
& +2 B_{1} C_{11}\left[\left(\frac{\beta_{1}}{\sigma_{D}}\right)^{2}+\left(\alpha_{s, 1} \sigma_{\mu, 1}\right)^{2}\right]+2 B_{2} C_{12}\left[\left(\frac{\beta_{2}}{\sigma_{D}}\right)^{2}+\left(\alpha_{s, 2} \sigma_{\mu, 2}\right)^{2}\right]+2 B_{3} C_{13} \sigma_{\mu}^{2} \\
& -\chi B_{1} \frac{\beta_{1}}{\sigma_{D}^{2}}-\chi B_{2} \frac{\beta_{2}}{\sigma_{D}^{2}}-\chi B_{3} \frac{\alpha_{D} \sigma_{\mu}}{\sigma_{D}} \\
& +2 B_{1} C_{12}\left[\frac{\beta_{1} \beta_{2}}{\sigma_{D}^{2}}+\alpha_{s, 1} \alpha_{s, 2} \sigma_{\mu, 1} \sigma_{\mu, 2}\right]+2 B_{2} C_{11}\left[\frac{\beta_{1} \beta_{2}}{\sigma_{D}^{2}}+\alpha_{s, 1} \alpha_{s, 2} \sigma_{\mu, 1} \sigma_{\mu, 2}\right] \\
& +2 B_{1} C_{13}\left[\frac{\beta_{1} \alpha_{D} \sigma_{\mu}}{\sigma_{D}}+\alpha_{s} \alpha_{s, 1} \sigma_{\mu} \sigma_{\mu, 1}\right]+2 B_{3} C_{11}\left[\frac{\beta_{1} \alpha_{D} \sigma_{\mu}}{\sigma_{D}}+\alpha_{s} \alpha_{s, 1} \sigma_{\mu} \sigma_{\mu, 1}\right] \\
& +2 B_{2} C_{13}\left[\frac{\beta_{2} \alpha_{D} \sigma_{\mu}}{\sigma_{D}}+\alpha_{s} \alpha_{s, 2} \sigma_{\mu} \sigma_{\mu, 2}\right]+2 B_{3} C_{12}\left[\frac{\beta_{2} \alpha_{D} \sigma_{\mu}}{\sigma_{D}}+\alpha_{s} \alpha_{s, 2} \sigma_{\mu} \sigma_{\mu, 2}\right]
\end{aligned}
$$




$$
\begin{aligned}
\frac{\partial B_{2}}{\partial \tau}= & 2 C_{12} \kappa_{1} \bar{\mu}_{1}-\left(\kappa_{2}+\frac{\beta_{2}}{\sigma_{D}^{2}}\right) B_{2}+2 C_{22} \kappa_{2} \bar{\mu}_{2}+2 C_{23} \kappa \bar{\mu} \\
& +2 B_{1} C_{12}\left[\left(\frac{\beta_{1}}{\sigma_{D}}\right)^{2}+\left(\alpha_{s, 1} \sigma_{\mu, 1}\right)^{2}\right]+2 B_{2} C_{22}\left[\left(\frac{\beta_{2}}{\sigma_{D}}\right)^{2}+\left(\alpha_{s, 2} \sigma_{\mu, 2}\right)^{2}\right]+2 B_{3} C_{23} \sigma_{\mu}^{2} \\
& +\chi B_{1} \frac{\beta_{1}}{\sigma_{D}^{2}}+\chi B_{2} \frac{\beta_{2}}{\sigma_{D}^{2}}+\chi B_{3} \frac{\alpha_{D} \sigma_{\mu}}{\sigma_{D}} \\
& +2 B_{1} C_{22}\left[\frac{\beta_{1} \beta_{2}}{\sigma_{D}^{2}}+\alpha_{s, 1} \alpha_{s, 2} \sigma_{\mu, 1} \sigma_{\mu, 2}\right]+2 B_{2} C_{12}\left[\frac{\beta_{1} \beta_{2}}{\sigma_{D}^{2}}+\alpha_{s, 1} \alpha_{s, 2} \sigma_{\mu, 1} \sigma_{\mu, 2}\right] \\
& +2 B_{1} C_{23}\left[\frac{\beta_{1} \alpha_{D} \sigma_{\mu}}{\sigma_{D}}+\alpha_{s} \alpha_{s, 1} \sigma_{\mu} \sigma_{\mu, 1}\right]+2 B_{3} C_{12}\left[\frac{\beta_{1} \alpha_{D} \sigma_{\mu}}{\sigma_{D}}+\alpha_{s} \alpha_{s, 1} \sigma_{\mu} \sigma_{\mu, 1}\right] \\
& +2 B_{2} C_{23}\left[\frac{\beta_{2} \alpha_{D} \sigma_{\mu}}{\sigma_{D}}+\alpha_{s} \alpha_{s, 2} \sigma_{\mu} \sigma_{\mu, 2}\right]+2 B_{3} C_{22}\left[\frac{\beta_{2} \alpha_{D} \sigma_{\mu}}{\sigma_{D}}+\alpha_{s} \alpha_{s, 2} \sigma_{\mu} \sigma_{\mu, 2}\right] \\
\frac{\partial B_{3}}{\partial \tau}= & B_{1} \frac{\beta_{1}}{\sigma_{D}^{2}}+B_{2} \frac{\beta_{2}}{\sigma_{D}^{2}}-B_{3} \kappa \\
& +2 C_{13} \kappa_{1} \bar{\mu}_{1}+2 C_{23} \kappa_{2} \bar{\mu}_{2}+2 C_{33} \kappa \bar{\mu} \\
& +2 B_{1} C_{13}\left[\left(\frac{\beta_{1}}{\sigma_{D}}\right)^{2}+\left(\alpha_{s, 1} \sigma_{\mu, 1}\right)^{2}\right]+2 B_{2} C_{23}\left[\left(\frac{\beta_{2}}{\sigma_{D}}\right)^{2}+\left(\alpha_{s, 2} \sigma_{\mu, 2}\right)^{2}\right]+2 B_{3} C_{33} \sigma_{\mu}^{2} \\
& +2 B_{1} C_{23}\left[\frac{\beta_{1} \beta_{2}}{\sigma_{D}^{2}}+\alpha_{s, 1} \alpha_{s, 2} \sigma_{\mu, 1} \sigma_{\mu, 2}\right]+2 B_{2} C_{13}\left[\frac{\beta_{1} \beta_{2}}{\sigma_{D}^{2}}+\alpha_{s, 1} \alpha_{s, 2} \sigma_{\mu, 1} \sigma_{\mu, 2}\right] \\
& +2 B_{1} C_{33}\left[\frac{\beta_{1} \alpha_{D} \sigma_{\mu}}{\sigma_{D}}+\alpha_{s} \alpha_{s, 1} \sigma_{\mu} \sigma_{\mu, 1}\right]+2 B_{3} C_{13}\left[\frac{\beta_{1} \alpha_{D} \sigma_{\mu}}{\sigma_{D}}+\alpha_{s} \alpha_{s, 1} \sigma_{\mu} \sigma_{\mu, 1}\right] \\
& +2 B_{2} C_{33}\left[\frac{\beta_{2} \alpha_{D} \sigma_{\mu}}{\sigma_{D}}+\alpha_{s} \alpha_{s, 2} \sigma_{\mu} \sigma_{\mu, 2}\right]+2 B_{3} C_{23}\left[\frac{\beta_{2} \alpha_{D} \sigma_{\mu}}{\sigma_{D}}+\alpha_{s} \alpha_{s, 2} \sigma_{\mu} \sigma_{\mu, 2}\right] \\
& +2 .
\end{aligned}
$$

Finally, for the constant terms one gets

$$
\begin{aligned}
\frac{\partial A}{\partial \tau}= & B_{1} \kappa_{1} \bar{\mu}_{1}+B_{2} \kappa_{2} \bar{\mu}_{2}+B_{3} \kappa \bar{\mu} \\
& +\left(\frac{1}{2} B_{1}^{2}+C_{11}\right)\left[\left(\frac{\beta_{1}}{\sigma_{D}}\right)^{2}+\left(\alpha_{s, 1} \sigma_{\mu, 1}\right)^{2}\right]+\left(\frac{1}{2} B_{2}^{2}+C_{22}\right)\left[\left(\frac{\beta_{2}}{\sigma_{D}}\right)^{2}+\left(\alpha_{s, 2} \sigma_{\mu, 2}\right)^{2}\right] \\
& +\left(\frac{1}{2} B_{3}^{2}+C_{33}\right) \sigma_{\mu}^{2} \\
& +2 C_{12}\left[\frac{\beta_{1} \beta_{2}}{\sigma_{D}^{2}}+\alpha_{s, 1} \alpha_{s, 2} \sigma_{\mu, 1} \sigma_{\mu, 2}\right]+B_{1} B_{2}\left[\frac{\beta_{1} \beta_{2}}{\sigma_{D}^{2}}+\alpha_{s, 1} \alpha_{s, 2} \sigma_{\mu, 1} \sigma_{\mu, 2}\right] \\
& +2 C_{13}\left[\frac{\beta_{1} \alpha_{D} \sigma_{\mu}}{\sigma_{D}}+\alpha_{s} \alpha_{s, 1} \sigma_{\mu} \sigma_{\mu, 1}\right]+B_{1} B_{3}\left[\frac{\beta_{1} \alpha_{D} \sigma_{\mu}}{\sigma_{D}}+\alpha_{s} \alpha_{s, 1} \sigma_{\mu} \sigma_{\mu, 1}\right] \\
& +2 C_{23}\left[\frac{\beta_{2} \alpha_{D} \sigma_{\mu}}{\sigma_{D}}+\alpha_{s} \alpha_{s, 2} \sigma_{\mu} \sigma_{\mu, 2}\right]+B_{2} B_{3}\left[\frac{\beta_{2} \alpha_{D} \sigma_{\mu}}{\sigma_{D}}+\alpha_{s} \alpha_{s, 2} \sigma_{\mu} \sigma_{\mu, 2}\right]
\end{aligned}
$$


Now collect the functions $C_{11}, \ldots, C_{33}$ in the matrix $C$ with

$$
C=\left(\begin{array}{lll}
C_{11} & C_{12} & C_{13} \\
C_{12} & C_{22} & C_{23} \\
C_{12} & C_{23} & C_{33}
\end{array}\right)
$$

and define the matrices $P, Q, R$ as follows:

$$
\begin{aligned}
P= & \left(\begin{array}{ccc}
-\left(\kappa_{1}+\frac{\beta_{1}}{\sigma_{D}^{2}}\right)-\chi \frac{\beta_{1}}{\sigma_{D}^{2}} & -\chi \frac{\beta_{2}}{\sigma_{D}^{2}} & -\chi \frac{\alpha_{D} \sigma_{\mu}}{\sigma_{D}} \\
\chi \frac{\beta_{1}}{\sigma_{D}^{2}} & -\left(\kappa_{2}+\frac{\beta_{2}}{\sigma_{D}^{2}}\right)+\chi \frac{\beta_{2}}{\sigma_{D}^{2}} & \chi \frac{\alpha_{D} \sigma_{\mu}}{\sigma_{D}} \\
\frac{\beta_{1}}{\sigma_{D}^{2}} & \frac{\beta_{2}}{\sigma_{D}^{2}} & -\kappa
\end{array}\right) \\
Q= & \frac{1}{2 \sigma_{D}^{2}}\left(\begin{array}{ccc}
\chi(\chi+1) & -\chi^{2} & -\chi \\
-\chi^{2} & \chi(\chi-1) & \chi \\
-\chi & \chi & 0
\end{array}\right) \\
R= & -2\left(\begin{array}{ccc}
\left(\frac{\beta_{1}}{\sigma_{D}}\right)^{2}+\left(\alpha_{s, 1} \sigma_{\mu, 1}\right)^{2} & \frac{\beta_{1} \beta_{2}}{\sigma_{D}^{2}}+\alpha_{s, 1} \alpha_{s, 2} \sigma_{\mu, 1} \sigma_{\mu, 2} & \frac{\beta_{1} \alpha_{D} \sigma_{\mu}}{\sigma_{D}}+\alpha_{s} \alpha_{s, 1} \sigma_{\mu} \sigma_{\mu, 1} \\
\frac{\beta_{1} \beta_{2}}{\sigma_{D}^{2}}+\alpha_{s, 1} \alpha_{s, 2} \sigma_{\mu, 1} \sigma_{\mu, 2} & \left(\frac{\beta_{2}}{\sigma_{D}}\right)^{2}+\left(\alpha_{s, 2} \sigma_{\mu, 2}\right)^{2} & \frac{\beta_{2} \alpha_{D} \sigma_{\mu}}{\sigma_{D}}+\alpha_{s} \alpha_{s, 2} \sigma_{\mu} \sigma_{\mu, 2} \\
\frac{\beta_{1} \alpha_{D} \sigma_{\mu}}{\sigma_{D}}+\alpha_{s} \alpha_{s, 1} \sigma_{\mu} \sigma_{\mu, 1} & \frac{\beta_{2} \alpha_{D} \sigma_{\mu}}{\sigma_{D}}+\alpha_{s} \alpha_{s, 2} \sigma_{\mu} \sigma_{\mu, 2} & \sigma_{\mu}^{2}
\end{array}\right)
\end{aligned}
$$

Then the matrix-valued function $C$ satisfy the matrix Ricatti differential equation

$$
\frac{\partial C}{\partial \tau}=P C+C P^{\prime}+Q-C R C
$$

with initial condition $C(0)=0$. The solution to this equation can be characterized via matrix exponentials, but we solve Appendix A.6 numerically. Next define the vector $\theta=\left(\kappa_{1} \bar{\mu}_{1}, \kappa_{2} \bar{\mu}_{2}, \kappa \bar{\mu}\right)^{\prime}$. Then the vector-valued function $B \equiv\left(B_{1}, B_{2}, B_{3}\right)^{\prime}$ satisfies the ordinary differential equation

$$
\frac{\partial B}{\partial \tau}=2 C \theta+(P-C R) B
$$

again with initial condition $B(0)=0$. We solve this equation numerically using a standard MATLAB solver for ordinary differential equations. Finally, the function $A$ satisfies

$$
\frac{\partial A}{\partial \tau}=B^{\prime} \theta+\frac{1}{2} \operatorname{trace}\left[\left(B B^{\prime}+2 C\right) \tilde{R}\right]
$$

where $\tilde{R}=-\frac{1}{2} R$ and $A(0)=0$. This equation can be solved by numerically integrating the expression on the right-hand side, since the functions $B$ and $C$ have been commuted in the previous steps. 
True parameters for dividend and growth rate processes

\begin{tabular}{lcc}
\hline Volatility of dividend growth rate & $\sigma_{D}$ & 0.13 \\
Volatility in the mean reversion process of dividend growth rate & $\sigma_{\mu}$ & 0.015 \\
Long run mean of dividend growth rate & $\bar{\mu}$ & 0.015 \\
Speed of mean reversion & $\kappa$ & 0.2 \\
Local correlation between signal and expected dividend growth & $\alpha_{s}$ & 0 \\
Local correlation between dividend and expected dividend growth & $\alpha_{D}$ & 0 \\
\hline
\end{tabular}

Basic parameter ranges in numerical analysis (before check of growth condition)

Local correlation between signal and expected dividend growth

$\alpha_{s} \in[-0.99 ; 0.99]$

Local correlation between dividend and expected dividend growth

$\alpha_{D} \in[-0.5 ; 0.5]$

Volatility in the mean reversion process of dividend growth rate

$\sigma_{\mu} \in[0.005 ; 0.055]$

Long run mean of dividend growth rate

$\bar{\mu} \in[0 ; 0.03]$

Speed of mean reversion

$\kappa \in[0.1 ; 0.3]$

Parameters for investors

Relative risk aversion of both groups 3

Ratio between both investors' initial wealth levels 1

Subjective discount rate

$\delta$

State variables

Change between subjective measures at time 0

$\eta_{0}$

Initial estimation errors at time 0

$m_{0,1}, m_{0,2}$

0

Table A.1: Values for Parameters and State Variables

The table shows the values for parameters and state variables used in the numerical analysis of our model. The true parameter values are taken from Dumas et al. (2009). 

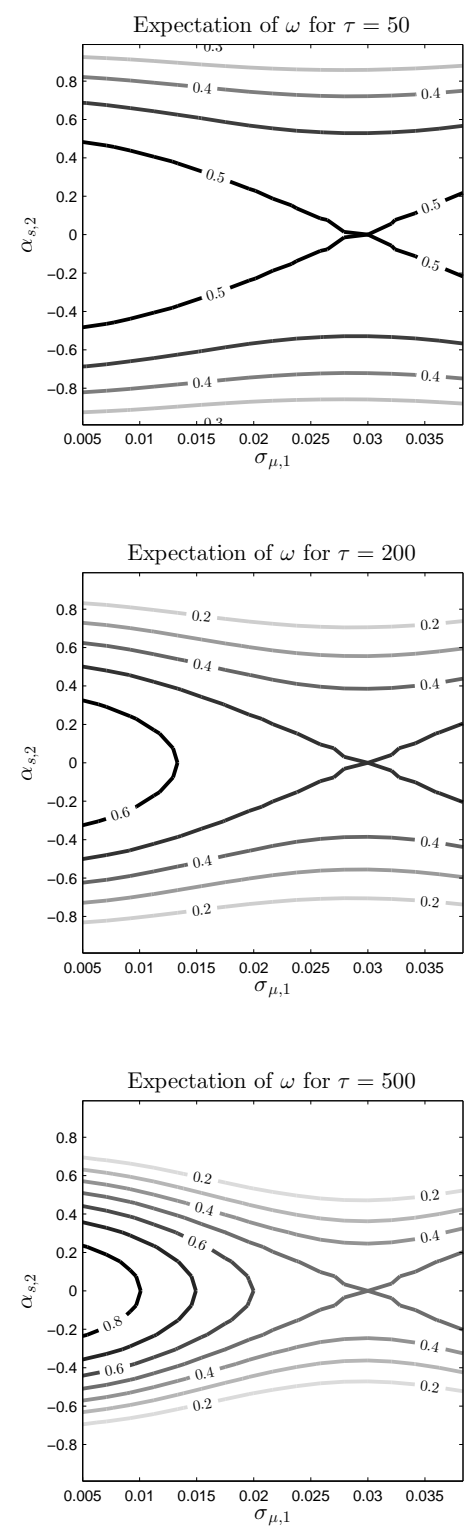

Figure A.1: Expected Consumption Shares for Combinations of $\sigma_{\mu, 1}$ and $\alpha_{s, 2}$

The plots show the contour lines for investor 2's expected consumption share $\omega$ after 50 , 200 , and 500 years. $\sigma_{\mu, 1}$ is the volatility of the process for the expected growth rate $\mu$ as assumed by investor $1 . \alpha_{s, 2}$ is the correlation assumed by investor 2 between signal innovations and innovations in $\mu$. The expected consumption share is computed as described in Appendix Appendix A.6. The parameters are shown in Table A.1. The plot ranges are restricted to the combinations of $\sigma_{\mu, 1}$ and $\alpha_{s, 2}$ satisfying the growth condition for the stock price in Equation Appendix A.5. 

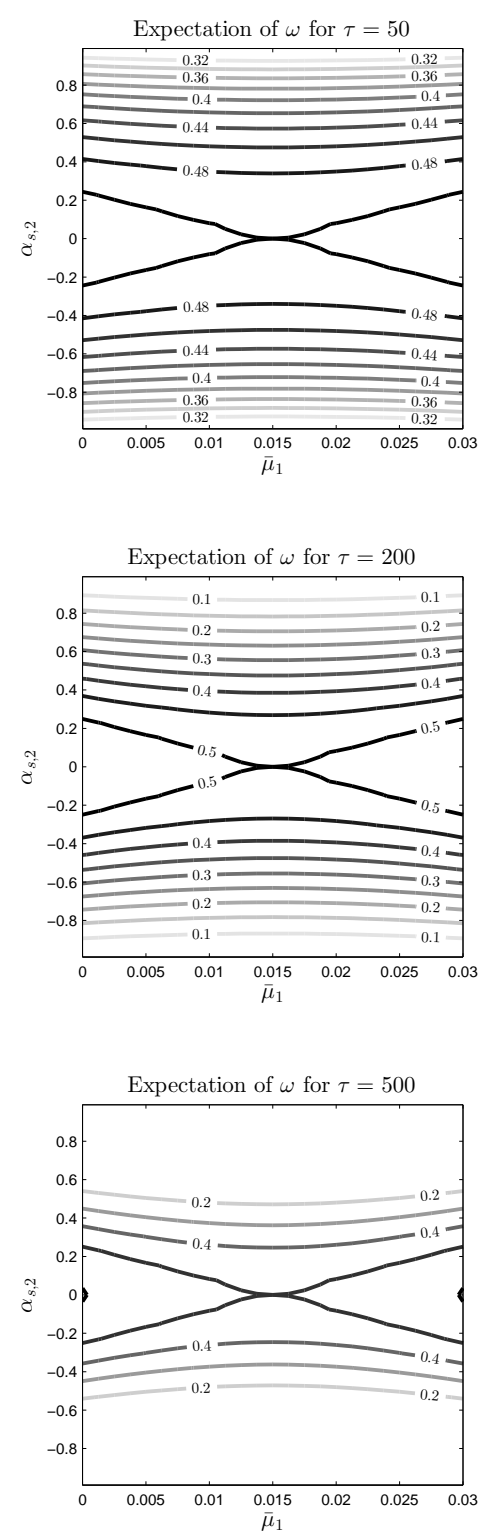

Figure A.2: Expected Consumption Shares for Combinations of $\bar{\mu}_{1}$ and $\alpha_{s, 2}$

The plots show the contour lines for investor 2's expected consumption share $\omega$ after 50,200 , and 500 years. $\bar{\mu}_{1}$ is long-run mean of the expected growth rate $\mu$ as assumed by investor $1 . \alpha_{s, 2}$ is the correlation assumed by investor 2 between signal innovations and innovations in $\mu$. The expected consumption share is computed as described in Appendix Appendix A.6. The parameters are shown in Table A.1. The plot ranges are restricted to the combinations of $\bar{\mu}_{1}$ and $\alpha_{s, 2}$ satisfying the growth condition for the stock price in Equation Appendix A.5. 

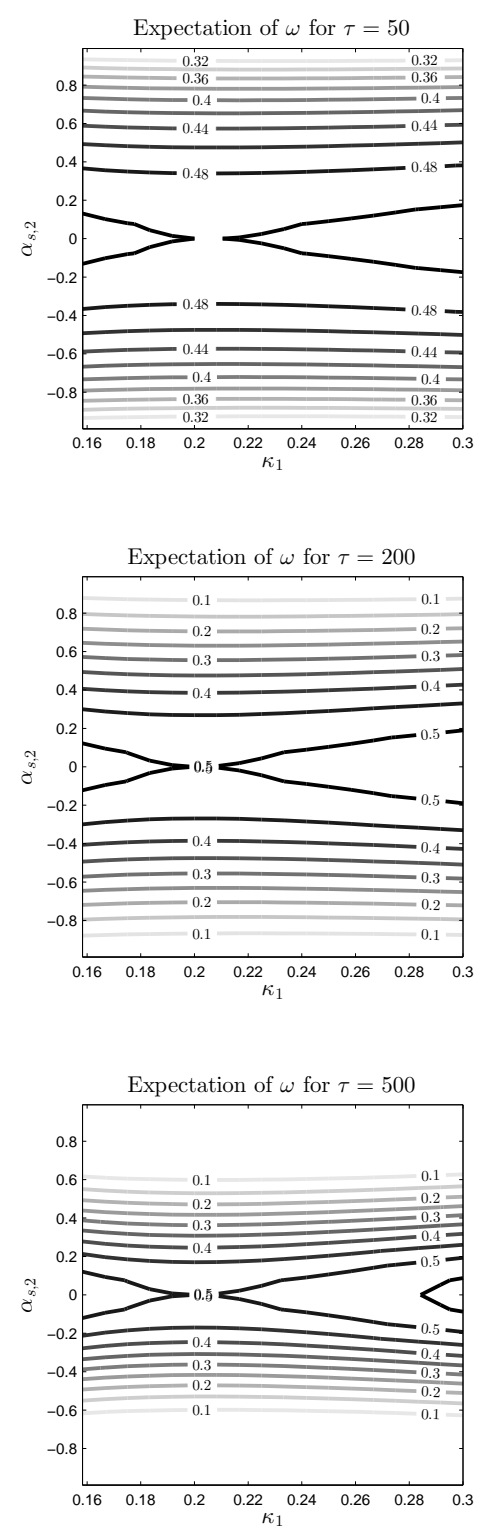

Figure A.3: Expected Consumption Shares for Combinations of $\kappa_{1}$ and $\alpha_{s, 2}$

The plots show the contour lines for investor 2's expected consumption share $\omega$ after 50,200 , and 500 years. $\kappa_{1}$ is the speed of mean reversion for the expected growth rate process assumed by investor 1. $\alpha_{s, 2}$ is the correlation assumed by investor 2 between signal innovations and innovations in $\mu$. The expected consumption share is computed as described in Appendix Appendix A.6. The parameters are shown in Table A.1. The plot ranges are restricted to the combinations of $\kappa_{1}$ and $\alpha_{s, 2}$ satisfying the growth condition for the stock price in Equation Appendix A.5. 

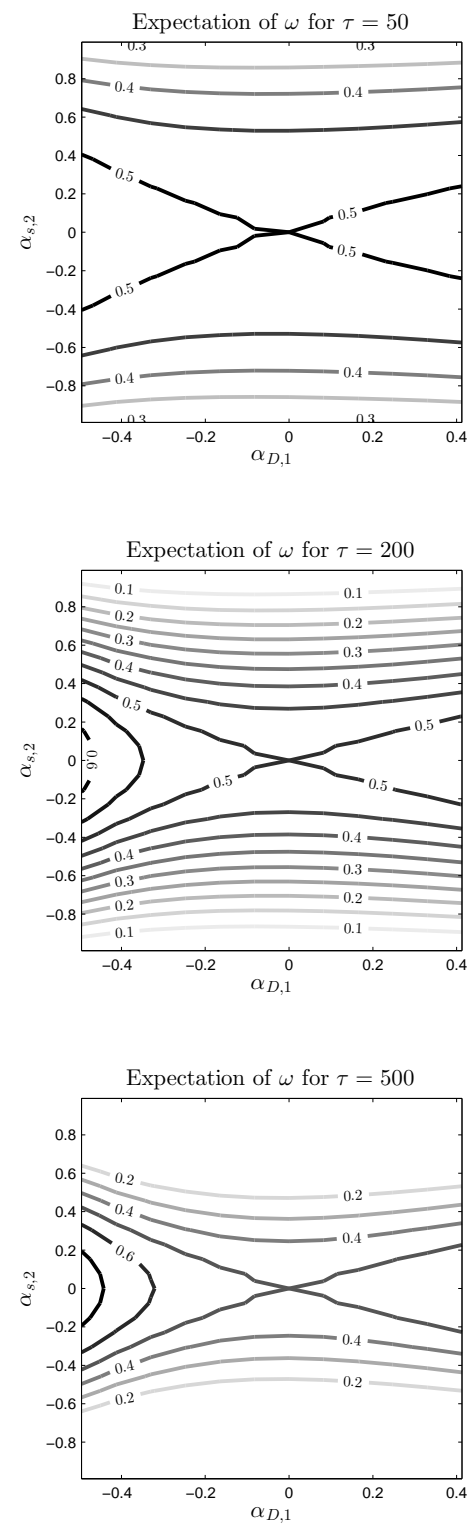

Figure A.4: Expected Consumption Shares for Combinations of $\alpha_{D, 1}$ and $\alpha_{s, 2}$

The plots show the contour lines for investor 2's expected consumption share $\omega$ after 50,200 , and 500 years. $\alpha_{D, 1}$ is the correlation assumed by investor 1 between dividend innovations and innovations to the expected growth rate $\mu . \alpha_{s, 2}$ is the correlation assumed by investor 2 between signal innovations and innovations in $\mu$. The expected consumption share is computed as described in Appendix Appendix A.6. The parameters are shown in Table A.1. The plot ranges are restricted to the combinations of $\alpha_{D, 1}$ and $\alpha_{s, 2}$ satisfying the growth condition for the stock price in Equation Appendix A.5. 

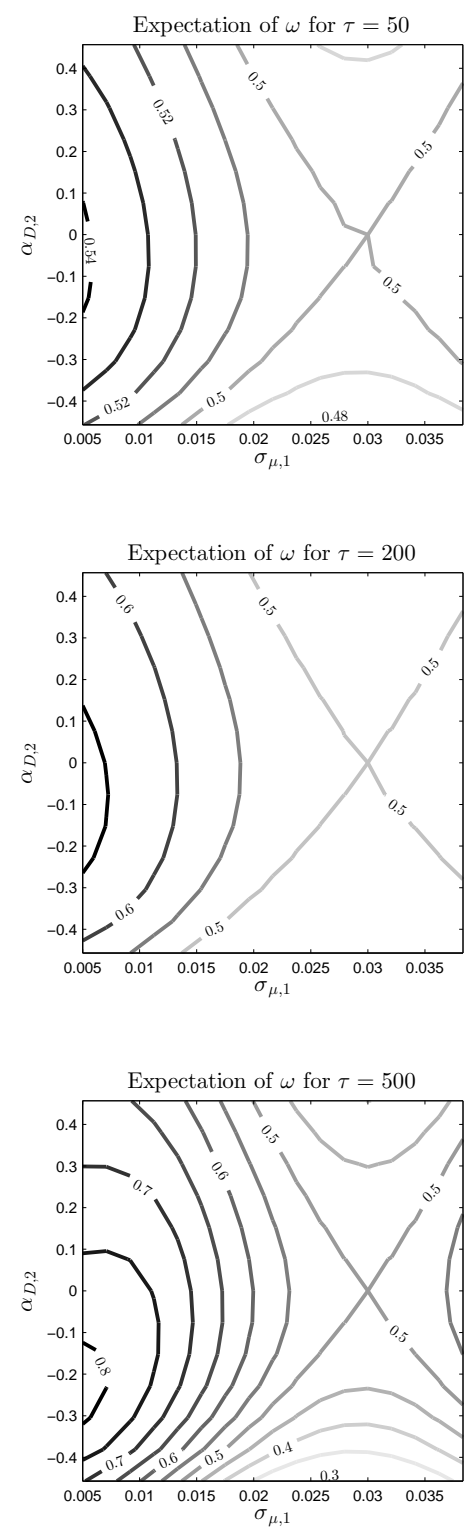

Figure A.5: Expected Consumption Shares for Combinations of $\sigma_{\mu, 1}$ and $\alpha_{D, 2}$

The plots show the contour lines for investor 2's expected consumption share $\omega$ after 50,200 , and 500 years. $\sigma \mu, 1$ is the volatility of the process for the expected growth rate $\mu$ as assumed by investor $1 . \alpha_{D, 2}$ is the correlation assumed by investor 2 between dividend innovations and innovations in $\mu$. The expected consumption share is computed as described in Appendix Appendix A.6. The parameters are shown in Table A.1. The plot ranges are restricted to the combinations of $\sigma_{\mu, 1}$ and $\alpha_{D, 2}$ satisfying the growth condition for the stock price in Equation Appendix A.5. 
Volatility Stock Price

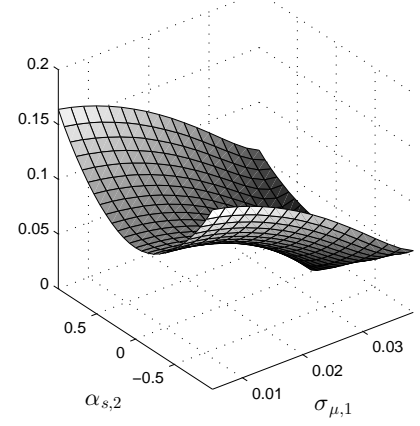

Volatility Wealth Investor 1

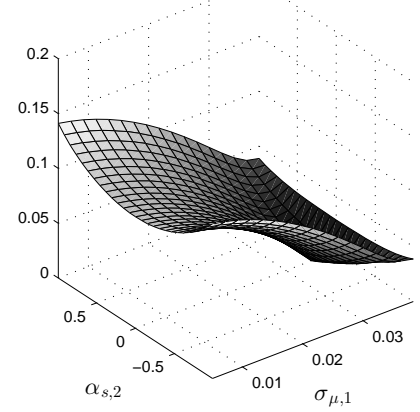

Volatility Wealth Investor 2

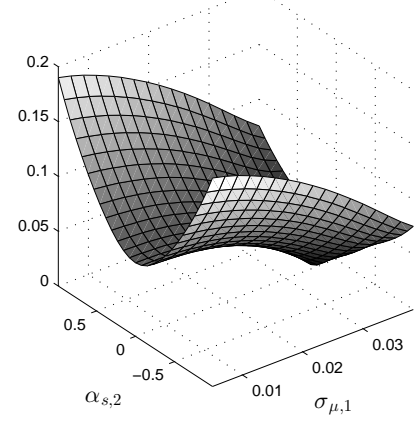

Expected Excess Return Stock

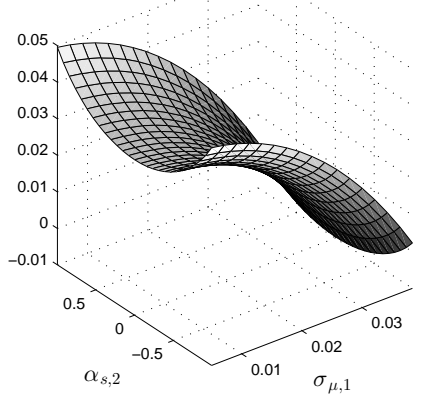

Expected Excess Return Wealth Investor 1

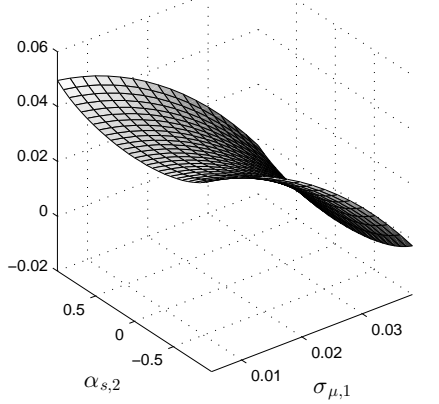

Expected Excess Return Wealth Investor 2

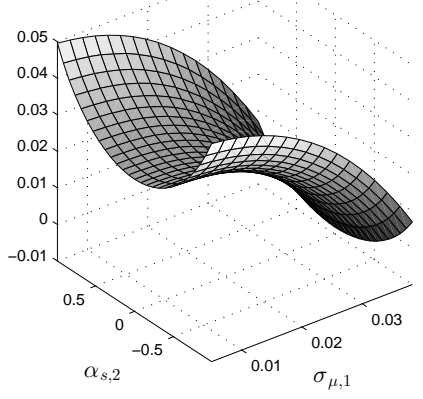

Figure A.6: Asset Pricing Moments for Combinations of $\sigma_{\mu, 1}$ and $\alpha_{s, 2}$

The plots show the expected excess return and the return volatility of the stock (corresponding to aggregate wealth) and the two investors' respective individual wealth levels. $\sigma_{\mu, 1}$ is the volatility of the process for the expected growth rate $\mu$ as assumed by investor 1 . $\alpha_{s, 2}$ is the correlation assumed by investor 2 between signal innovations and innovations in $\mu$. The expected consumption share is computed as described in Appendix Appendix A.6. The parameters are shown in Table A.1. The plot ranges are restricted to the combinations of $\sigma_{\mu, 1}$ and $\alpha_{s, 2}$ satisfying the growth condition for the stock price in Equation Appendix A.5. 

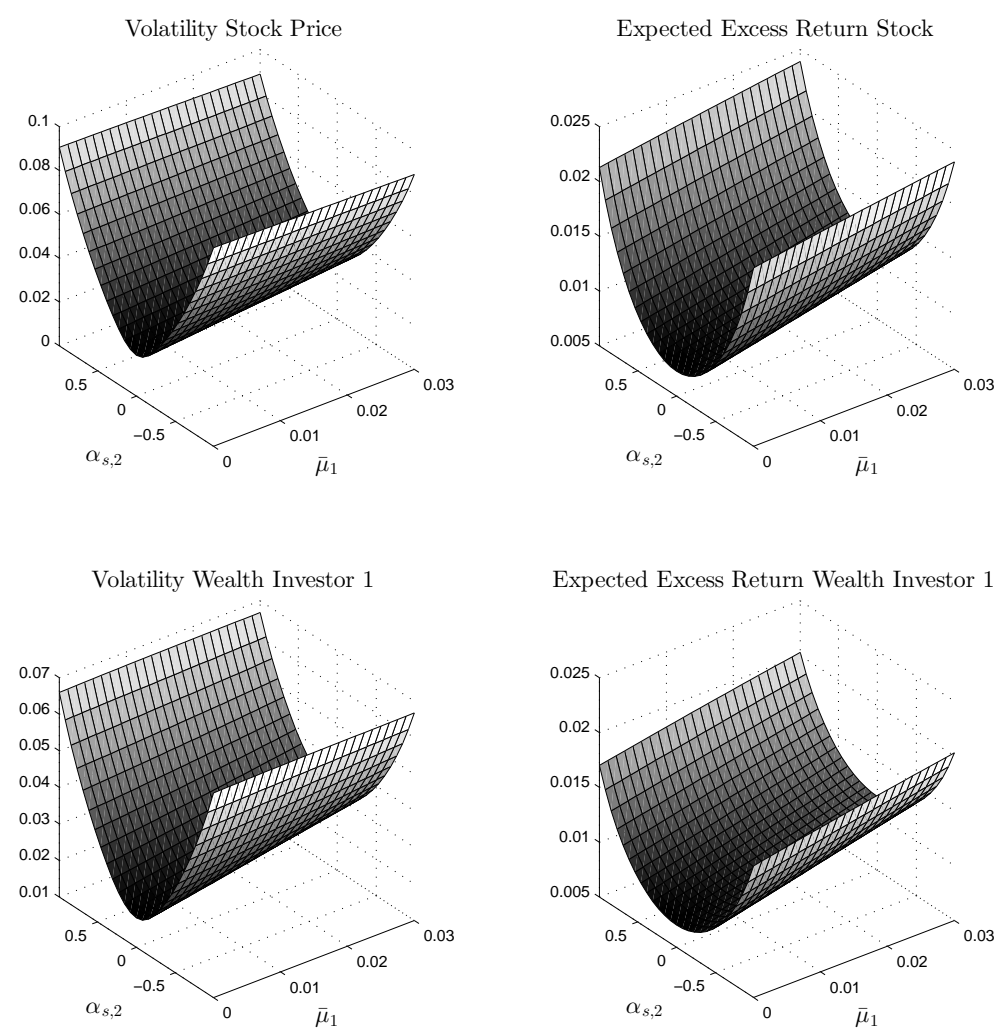

Volatility Wealth Investor 2

Expected Excess Return Wealth Investor 2
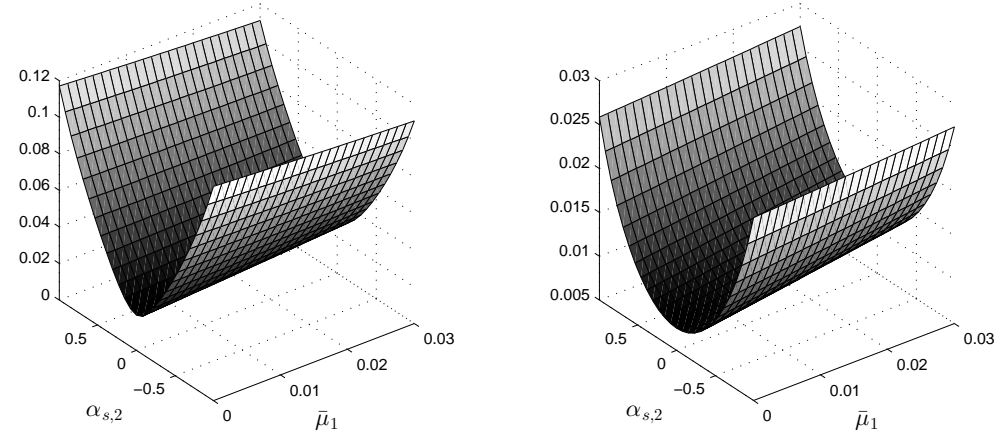

Figure A.7: Asset Pricing Moments for Combinations of $\bar{\mu}_{1}$ and $\alpha_{s, 2}$

The plots show the expected excess return and the return volatility of the stock (corresponding to aggregate wealth) and the two investors' respective individual wealth levels. $\bar{\mu}_{1}$ is long-run mean of the expected growth rate $\mu$ as assumed by investor $1 . \alpha_{s, 2}$ is the correlation assumed by investor 2 between signal innovations and innovations in $\mu$. The expected consumption share is computed as described in Appendix Appendix A.6. The parameters are shown in Table A.1. The plot ranges are restricted to the combinations of $\bar{\mu}_{1}$ and $\alpha_{s, 2}$ satisfying the growth condition for the stock price in Equation Appendix A.5. 

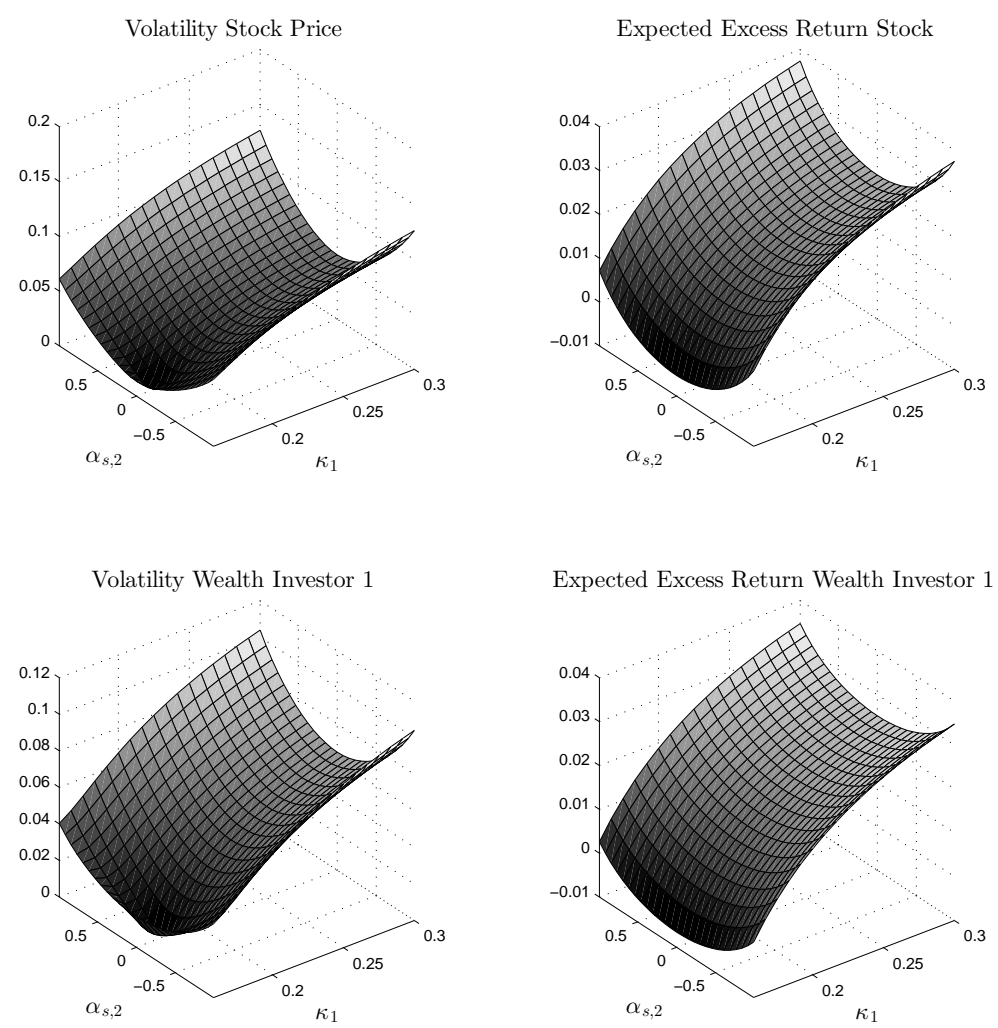

Volatility Wealth Investor 2

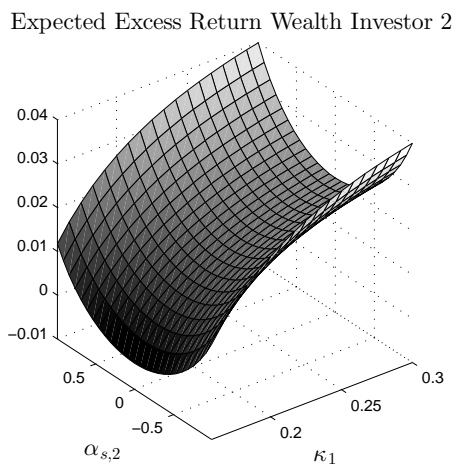

Figure A.8: Asset Pricing Moments for Combinations of $\kappa_{1}$ and $\alpha_{s, 2}$

The plots show the expected excess return and the return volatility of the stock (corresponding to aggregate wealth) and the two investors' respective individual wealth levels. $\kappa_{1}$ is the speed of mean reversion for the expected growth rate process assumed by investor 1 . $\alpha_{s, 2}$ is the correlation assumed by investor 2 between signal innovations and innovations in $\mu$. The expected consumption share is computed as described in Appendix Appendix A.6. The parameters are shown in Table A.1. The plot ranges are restricted to the combinations of $\kappa_{1}$ and $\alpha_{s, 2}$ satisfying the growth condition for the stock price in Equation Appendix A.5. 
Volatility Stock Price

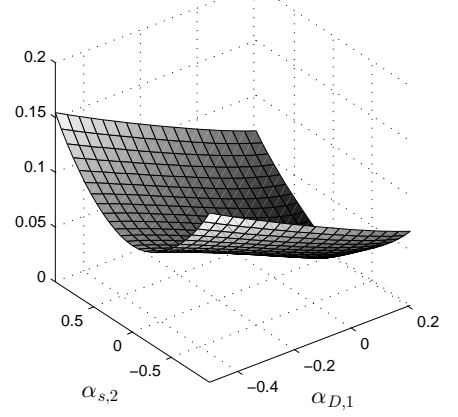

Volatility Wealth Investor 1

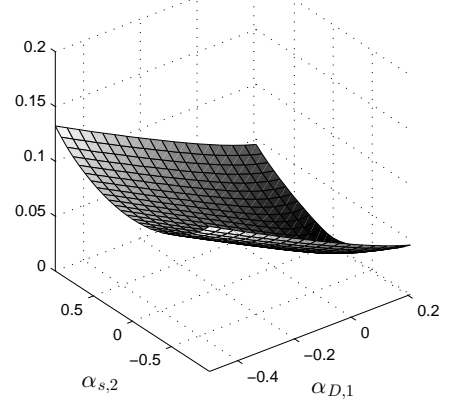

Volatility Wealth Investor 2

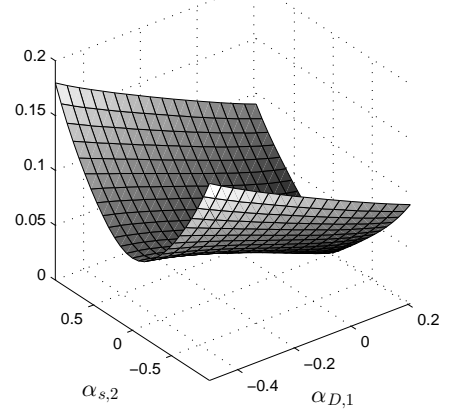

Expected Excess Return Stock

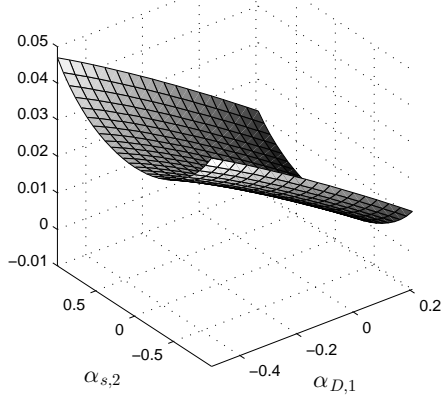

Expected Excess Return Wealth Investor 1

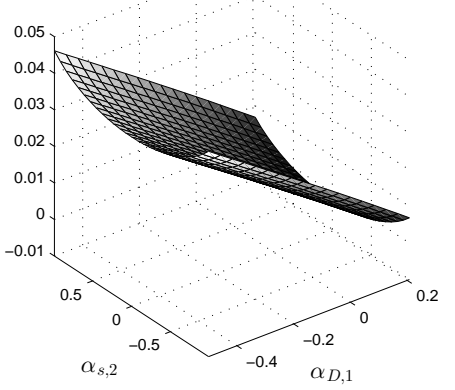

Expected Excess Return Wealth Investor 2

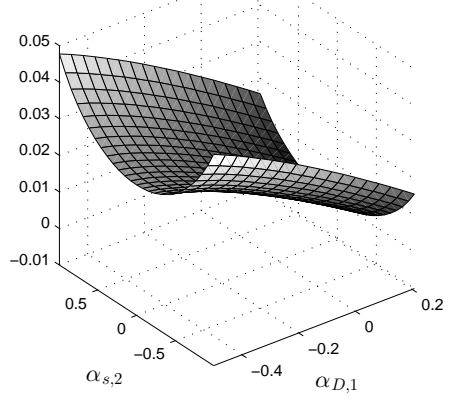

Figure A.9: Asset Pricing Moments for Combinations of $\alpha_{D, 1}$ and $\alpha_{s, 2}$

The plots show the expected excess return and the return volatility of the stock (corresponding to aggregate wealth) and the two investors' respective individual wealth levels. $\alpha_{D, 1}$ is the correlation assumed by investor 1 between dividend innovations and innovations to the expected growth rate $\mu . \alpha_{s, 2}$ is the correlation assumed by investor 2 between signal innovations and innovations in $\mu$. The expected consumption share is computed as described in Appendix Appendix A.6. The parameters are shown in Table A.1. The plot ranges are restricted to the combinations of $\alpha_{D, 1}$ and $\alpha_{s, 2}$ satisfying the growth condition for the stock price in Equation Appendix A.5. 
Volatility Stock Price

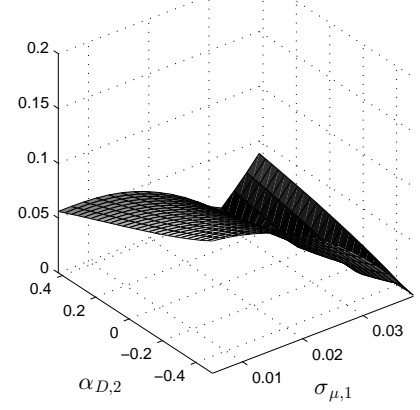

Volatility Wealth Investor 1

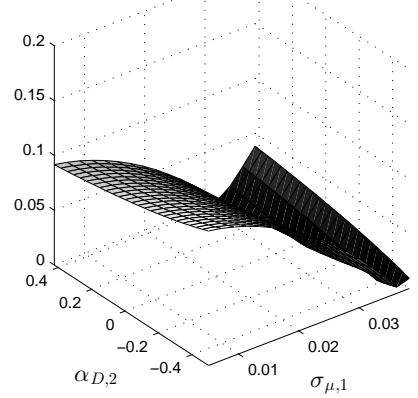

Volatility Wealth Investor 2

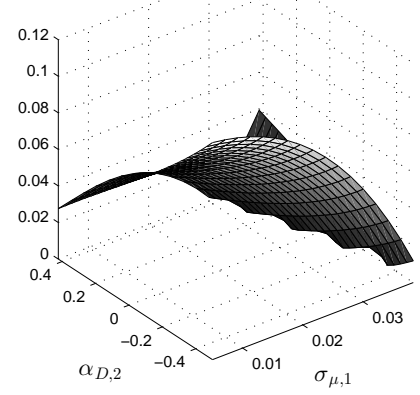

Expected Excess Return Stock

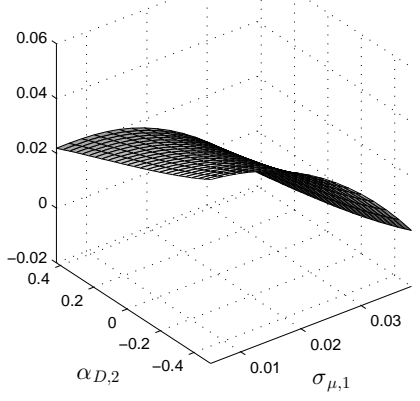

Expected Excess Return Wealth Investor 1

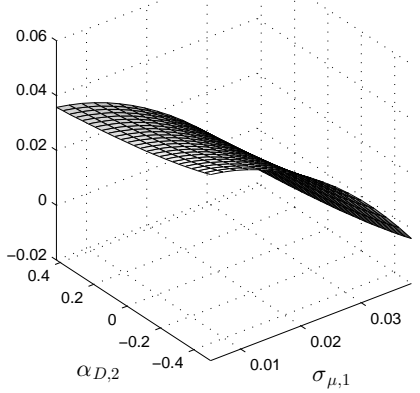

Expected Excess Return Wealth Investor 2

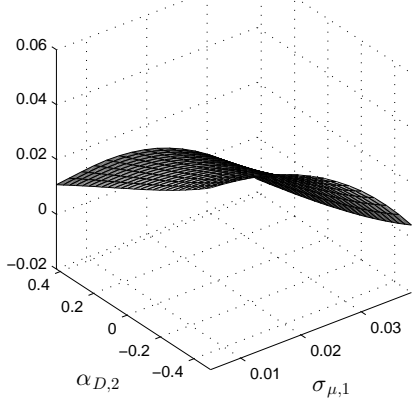

Figure A.10: Asset Pricing Moments for Combinations of $\sigma_{\mu, 1}$ and $\alpha_{D, 2}$

The plots show the expected excess return and the return volatility of the stock (corresponding to aggregate wealth) and the two investors' respective individual wealth levels. $\sigma_{\mu, 1}$ is the volatility of the process for the expected growth rate $\mu$ as assumed by investor 1. $\alpha_{D, 2}$ is the correlation assumed by investor 2 between dividend innovations and innovations in $\mu$. The expected consumption share is computed as described in Appendix Appendix A.6. The parameters are shown in Table A.1. The plot ranges are restricted to the combinations of $\sigma_{\mu, 1}$ and $\alpha_{D, 2}$ satisfying the growth condition for the stock price in Equation Appendix A.5. 


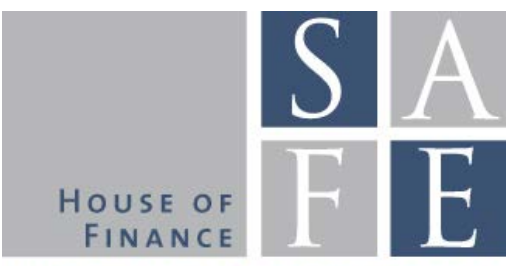

WORKING PAPER SERIES

\section{Recent Issues}

No. 113 Bettina Brüggemann, Jinhyuk Yoo

No. 112 Shafik Hebous, Alfons J.

Weichenrieder

No.111 Alfons J. Weichenrieder, Fangying $\mathrm{Xu}$

No. 110 Dirk Krueger, Alexander Ludwig

No.109 Tobias Tröger

No. 108 Sascha Baghestanian, Paul Gortner, Baptiste Massenot

No. 107 Daniel Powell, Marc Steffen Rapp

No. 106 Baptiste Massenot, Stéphane Straub

No. 105

No. 104

No. 103

Massimiliano Caporin, Loriana

Pelizzon, Francesco Ravazzolo, Roberto Rigobon

No. 102 Iñaki Aldasoro, Iván Alves

No.101 Marcel Grupp

No. $100 \quad$ Marcel Grupp
Aggregate and Distributional Effects of Increasing Taxes on Top Income Earners

On Deficits and Symmetries in a Fiscal Capacity

Are Tax Havens Good? Implications of the Crackdown on Secrecy

On the Optimal Provision of Social Insurance

Regulatory Influence on Market Conditions in the Banking Union

Compensation Schemes, Liquidity Provision, and Asset Prices: An Experimental Analysis

Non-Mandatory Say on Pay Votes and AGM Participation: Evidence from Germany

Informal Sector and Economic Development: The Credit Supply Channel

A Decentralization Theorem of Taxation

Predictably Irrational: Gambling for Resurrection in Experimental Asset Markets?

Measuring Sovereign Contagion in Europe

Multiplex interbank networks and systemic importance: An application to European data

On the Impact of Leveraged Buyouts on Bank Systemic Risk

Taking the Lead: When Non-Banks Arrange Syndicated Loans 